\title{
THE GENERALIZED MARTIN'S MINIMUM PROBLEM AND ITS APPLICATIONS IN SEVERAL COMPLEX VARIABLES
}

\author{
BY \\ SIIOZO MATSUURA
}

\begin{abstract}
The objectives of this paper are to generalize the Martin's $\mathfrak{L}^{2}$-minimum problem under more general additional conditions given by bounded linear functionals in a bounded domain $D$ in $C^{n}$ and to apply this problem to various directions.

We firstly define the new $i$ th biholomorphically invariant Kähler metric and the $i$ th representative domain $(i=0,1,2, \ldots)$, and secondly give estimates on curvatures with respect to the Bergman metric and investigate the asymptotic behaviors via an $A$-approach on the curvatures about a boundary point having a sort of pseudoconvexity.

Further, we study (i) the extensions of some results recently obtained by K. Kikuchi on the Ricci scalar curvature, (ii) a minimum property on the reproducing subspace-kernel in $\mathscr{L}_{(m)}^{2}(D)$, and (iii) an extension of the fundamental theorem of K. H. Look.
\end{abstract}

1. Introduction. The Bergman's minimum problem [3] with respect to $\mathfrak{L}^{2}(D)$ under some additional conditions has been extended by W. T. Martin [15] as the following (originally posed by W. Wirtinger [21]): Find the function $f(z)$ (belonging to $\mathscr{\complement}^{2}(D)$ or $\mathfrak{L}_{X, t}^{2}(D)$ ) which minimizes the Lebesgue square integral $(Q-f, Q-f)_{D}$ for a given function $Q(z, \bar{z}) \in L^{2}(D)$. Here $L^{2}(D)$ and $\varrho^{2}(D)$ denote the classes of square integrable and of square integrable holomorphic functions in a bounded domain $D$, respectively. $\mathcal{L}_{X, t}^{2}(D)$ denotes the class $\left\{f(z) \in \mathscr{L}^{2}(D) \mid f(t)=X, t \in D\right\}$.

In $\S 3$, under more general additional conditions using bounded linear functionals we shall get the generalized Martin's the orem, which includes the cases of Bergman [3], Martin [15] and others [17], [19], [20].

As an application of the minimum problem, in $\$ 4$ we shall define the

Received by the editors March 19, 1974。

AMS (MOS) subject classifications (1970). Primary 32H05, 32H10, 32H15; Secondary 53A55.

Key words and phrases. Bergman kernel function, Bergman metric, biholomorphically invariant, representative domain, bounded linear functional, holomorphic bisectional curvature, Ricci tensor, strictly pseudoconvex, classical Cartan domains. 
interesting quantities $\Omega_{D}^{(i)}(z)$ and $\widetilde{\Omega}_{D}^{(i)}(z)(i=0,1,2, \ldots)$ which have a sort of positivity and play important roles throughout this paper. Using these, we shall define the new $i$ th biholomorphically invariant Kähler metric $\left(d s_{D}^{(i)}\right)^{2}=\partial_{z}^{*} \partial_{z} \log \operatorname{det} \widetilde{K}_{D}^{(i)}(z, \bar{z})$ and the $i$ th representative domain $(i=$ $0,1,2, \ldots)$, where the biholomorphically relative invariants $\widetilde{K}_{D}^{(i)}(z, \bar{z})$ $(i=0,1,2, \ldots)$ are constructed by the Bergman kernel function of a bounded domain $D$ and its derivatives. In particular, $\left(d s_{D}^{(0)}\right)^{2}$ and $\left(d s_{D}^{(1)}\right)^{2}$ coincide with the Bergman metric [3] and the Fuks metric [8], respectively, and the Oth representative domain coincides with the Bergman representative domain.

In $\$ \$ 5,6$ and 7 , using the results of $\$ \S 3$ and 4 , we shall give various estimations (Theorems 5.1 and 5.2) on the holomorphic "bisectional" curvature $R_{D}(z ; u, v)$, the Ricci curvature $C_{D}(z ; u)$ and the Ricci scalar curvature $S_{D}(z)$ of a bounded domain $D$ with respect to the Bergman metric and generalize the results obtained by S. Bergman [1], [2], [3], B. A. Fuks [6], [7], [8] and others. For our purpose, the quantity $\Omega_{D}^{(2)}(z)$ and "the method of minimum integral" [3], [7] are used effectively.

In the case of $C^{2}$, the asymptotic behaviors of the Berginan kernel function $k_{D}(z, \bar{z})$ and related biholomorphic invariants about a boundary point $Q$ of a domain $D$ such that the Levi determinant $L(\phi)$ is positive at $Q$ have been studied minutely by S. Bergman [1] and B. A. Fuks [6], [7], L8]. But in the case of $C^{n}(n \geq 3)$, few results are known (see Chalmers [4], Hörmander [9]). On the asymptotic behaviors of the curvatures of a bounded domain $D$ in $C^{n}$ about a boundary point $Q$ at which $D$ is strictly pseudoconvex globally representable [4] and has the normal analytic hypersurface $h$ (through Q) lying entirely outside itself, in $\$ 7$ we shall prove that, using a sort of domains of comparison due to B. Chalmers [4], $R_{D}(z ; u)\left(\equiv R_{D}(z ; u, v)\right)$, $C_{D}(z ; u)$ and $S_{D}(z)$ tend to $-2 /(n+1),-1$ and $-n$ via an $A$-approach: $z \rightarrow Q$, respectively.

In $\S 8$, some results recently obtained by $\mathrm{K}$. Kikuchi L12] with respect to the Ricci scalar curvature as an application of the theorem of E. Hopf are extended.

In $\$ 9$, using the minimum problem with the condition that $Q(z, \bar{z}) \equiv$ $Q(z)=k_{D}(z, \bar{t}) \in \mathfrak{L}^{2}(D)$, where $k_{D}(z, \bar{t})$ denotes the Bergman kernel function of $D$, we shall show that the reproducing kernel function of a subspace $\mathfrak{L}_{(m)}^{2}(D)$ of $\mathfrak{L}^{2}(D)$ (see [S], L18]) has a sort of minimum property and give another expression of this kernel given in [5].

Finally, in $\$ 10$ a neat proof and an extension of the fundamental theorem (I) of K. H. Look [14] are given. 
2. Preliminaries. Throughout this paper we shall use, as far as possible, matrix representations, which give us available perspectives. For a matrix $A, \bar{A}, A^{T}$ and $A^{*}$ denote the conjugate, the transposed and the conjugate transposed matrices of $A$, respectively. The symbol $\times$ shows the Kronecker product and $[A]^{k}$ denotes $A \times \cdots \times A$ (k-times).

Let $D$ be a bounded schlicht domain in $C^{n}$ and $z \equiv\left(z_{1}, \ldots, z_{n}\right)^{T}$ be a complex $n \times 1$ vector variable in $D$. For the differential operator $D_{z} \equiv$ $\partial / \partial z \equiv\left(\partial / \partial z_{1}, \ldots, \partial / \partial z_{n}\right)\left(D_{z}^{*} \equiv \partial / \partial z^{*} \equiv(\partial / \partial \bar{z})^{T}\right)$, we shall define two sorts of the $k$ th order differential operators with respect to $z$ as follows:

$$
\left[D_{z}\right]^{k} \equiv[\partial / \partial z]^{k} \equiv(\partial / \partial z) \times \cdots \times(\partial / \partial z) \quad\left(1 \times n^{k} \text { vector }\right)
$$

and its contraction

$$
\begin{aligned}
D_{z}^{k} & \equiv \partial^{k} / \partial z^{k} \\
& \equiv\left(\partial^{k} / \partial z_{1}^{k}, \ldots,\left(k ! / k_{1} ! \cdots k_{n} !\right) \partial^{k} / \partial z_{1}^{k_{1}} \cdots \partial z_{n}^{k_{n}}, \ldots, \partial^{k} / \partial z_{n}^{k}\right)
\end{aligned}
$$

( $1 \times{ }_{n} H_{k}$ vector), where $\sum_{j=1}^{n} k_{j}=k$ and the arrangement of $\left\{k_{1}, \ldots, k_{n}\right\}$ is lexicographical. Using these operators, the $k$ th order derivatives of a matrix function $F(z, \bar{z}) \equiv\left(f_{p q}(z, \bar{z})\right)$ with respect to $z$ are defined by

$$
\left[D_{z}\right]^{k} F(z, \bar{z}) \equiv\left[D_{z}\right]^{k} \times F(z, \bar{z}) \equiv\left(\left[D_{z}\right]^{k} \times f_{p q}(z, \bar{z})\right)
$$

and

$$
D_{z}^{k} F(z, \bar{z}) \equiv D_{z}^{k} \times F(z, \bar{z}) \equiv\left(D_{z}^{k} \times f_{p q}(z, \bar{z})\right) .
$$

If we define the contracted $k$ th power of an $n \times 1$ vector $u \equiv\left(u_{1}, \ldots, u_{n}\right)^{T}$ as

$$
u^{k} \equiv\left(u_{1}^{k}, \ldots, u_{1}^{k_{1}} \ldots u_{n}^{k_{n}}, \ldots, u_{n}^{k}\right)^{T}
$$

it holds that, for a scalar function $f(z, \bar{z})$,

$$
\left(D_{z}^{k} f(z, \bar{z})\right) u^{k}=\left(\left[D_{z}\right]^{k} f(z, \bar{z})\right)[u]^{k}
$$

The total differential of a matrix function $F(z, \bar{z})(r \times s$ type $)$ is defined by

$$
d F(z, \bar{z}) \equiv \partial_{z} F+\partial_{z}^{*} F \equiv\left(D_{z} F\right)\left(d z \times E_{s}\right)+\left(d z^{*} \times E_{r}\right)\left(D_{z}^{*} F\right),
$$

where $d z \equiv\left(d z_{1}, \ldots, d z_{n}\right)^{T}$ and $E_{k}$ denotes the $k \times k$ unit matrix. 
In the following, we shall use some available formulas with respect to matrices, derivatives and differentials without proof [12], [16], [17]:

$$
D_{z}(A B)=\left(D_{z} A\right)\left(E_{n} \times B\right)+A\left(D_{z} B\right)
$$

( $A, B$ are $k \times l, l \times m$ matrices, respectively),

$$
D_{z}(A \times B)=\left(D_{z} A\right) \times B+\left(A \times D_{z} B\right)\left(\tilde{E}_{l n} \times E_{q}\right)
$$

$(A, B$ are $k \times l, p \times q$ matrices respectively and

$$
\widetilde{E}_{l n}=\left(\begin{array}{ccc}
e_{11} & \cdots & e_{l 1} \\
\vdots & & \vdots \\
e_{1 n} & \cdots & e_{l n}
\end{array}\right) \text {, }
$$

where $e_{i j}(i=1, \ldots, l ; j=1, \ldots, n)$ is an $l \times n$ matrix which has 1 as $(i, j)$-element and 0 's elsewhere),

$$
\begin{aligned}
& \partial_{z}\left(A^{-1}\right)=-A^{-1}\left(\partial_{z} A\right) A^{-1}=-A^{-1}\left(D_{z} A\right)\left(d z \times A^{-1}\right), \\
& D_{z}\left(A^{-1}\right)=-A^{-1}\left(D_{z} A\right)\left(E_{n} \times A^{-1}\right)
\end{aligned}
$$

( $A$ is a $k \times k$ regular matrix) and

$$
\partial_{z} \log \operatorname{det} A=\operatorname{Sp}\left(A^{-1} \partial_{z} A\right)=\operatorname{Sp}\left\{A^{-1}\left(D_{z} A\right)\left(d z \times E_{k}\right)\right\}
$$

( $A$ is a $k \times k$ regular matrix and $\mathrm{Sp}$ denotes the trace symbol). By (2.3) and (2.4) we have the following lemma.

Lemma 2.1. For a $k \times k$ regular matrix function $A(z, \bar{z})$ we bave

$$
\partial_{z}^{*} \partial_{z} \log \operatorname{det} A=\operatorname{Sp}\left\{\left(d z^{*} \times E_{k}\right)\left(A_{11}-A_{10} A^{-1} A_{01}\right)\left(d z \times E_{k}\right) A^{-1}\right\},
$$
where $A_{11}$ denotes $D_{z}^{*} D_{z} A$, etc.

Let $H(D)$ be the class of holomorphic matrix functions of all types in $D$ and $B H(D)$ be the subclass of $H(D)$ defined by

$$
B H(D) \equiv\left\{f(z) \equiv\left(f_{1}(z), \ldots, f_{n}(z)\right)^{T} \in H(D) \mid J_{f}(z) \neq 0 \text { in } D \subset C^{n}\right\},
$$

where $J_{f}(z)$ denotes the Jacobian determinant $\operatorname{det}(d f(z) / d z)\left(\equiv \operatorname{det}\left(D_{z} f(z)\right)\right)$. We call each-element belonging to $B H(D)$ a biholomorphic mapping, which is locally one-to-one in $D$. The subclass $\mathfrak{L}^{2}(D)$ of $H(D)$, which denotes the class of square Lebesgue integrable holomorphic functions in a bounded 
domain $D$, makes a complete Hilbert space with the Bergman reproducing kernel function $k_{D}(z, \bar{t})$.

3. General minimum problem. In this section, we shall generalize the results of S. Bergman [3], W. Wirtinger [21], W. T. Martin [15] and others for a given complex-valued $r \times 1$ vector function $Q(z, \bar{z}) \in L^{2}(D)$ and a general class (with more general additional conditions)

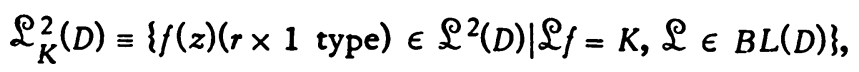

where $B L(D)$ denotes the class of all types of bounded linear functional matrices (see [S]) and $K$ denotes a given constant matrix of the same type as $\mathfrak{L}$.

Theorem 3.1. For a given $r \times 1$ vector function $Q(z, \bar{z}) \in L^{2}(D)$ in a bounded domain $D$, the minimizing function $M_{D, Q^{K}}^{K}(z) \in \mathcal{L}_{K}^{2}(D)$, which minimizes the Lekesgue square integral

$$
I(Q, f) \equiv(Q-f, Q-f)_{D} \equiv \operatorname{Sp} \int_{D}(Q(\zeta, \bar{\zeta})-f(\zeta))(Q(\zeta, \bar{\zeta})-f(\zeta)){ }^{*} \omega_{\zeta}
$$

under an additional condition

$$
\mathfrak{L}=K(K: \text { a given constant matrix, } \mathfrak{L} \in B L(D))
$$

with the condition $\operatorname{det}\left(\Phi^{*} \Phi\right) \neq 0$ for $\Phi \equiv \mathscr{L} \phi_{D}\left(\phi_{D}(z) \equiv\left(\phi_{1}(z), \phi_{2}(z), \ldots\right)^{T}\right.$ : an orthonormal system in $\left.\mathfrak{\complement}^{2}(D)\right)$, is given by

$$
M_{D, Q}^{K}(z)=\left\{B+(K-B \Phi)\left(\Phi^{*} \Phi\right)^{-1} \Phi^{*}\right\} \phi_{D}(z) \in \stackrel{2}{K}_{K}^{2}(D)
$$

and also the minimum value of $I(Q, f)$ is given by

$$
\lambda_{D, Q}^{K}=\operatorname{Sp}\left\{B B^{*}-B \Phi\left(\Phi^{*} \Phi\right)^{-1} \Phi^{*} B^{*}+K\left(\Phi^{*} \Phi\right)^{-1} K^{*}\right\},
$$

where $\omega_{\zeta}$ denotes the Euclidean volume element $\Pi_{j=1}^{n} d \bar{\zeta}_{j} \wedge d \zeta_{j} /(2 \sqrt{-1})^{n}$ and

$$
B \equiv\left(b_{i j}\right)=\int_{D} 2(\zeta, \bar{\zeta}) \phi_{D}^{*}(\zeta) \omega_{\zeta}
$$

Proof. Given a sufficiently large real number $M$, we consider a class $G \equiv\left\{\left.f(z) \in \mathfrak{L}_{K}^{2}(D)\left|\int_{D}\right| f(z)\right|^{2} \omega_{z} \leq M<+\infty\right\}$. $G$ becomes a compact family, and it is known that there exists a minimizing function $M_{D}^{K}(z) \equiv M_{D, Q=0}^{K}(z) \epsilon$ 
$\mathscr{L}_{K}^{2}(D)$ which minimizes the integral $I(0, f)=\int_{D}|f(z)|^{2} \omega_{z}$, where $M_{D}^{K}(z)$ is given by $K\left(\Phi^{*} \Phi\right)^{-1} \Phi^{*} \phi_{D}(z)$ and $\operatorname{det}\left(\Phi^{*} \Phi\right) \neq 0$ (see [3]).

Now, we will follow the procedure of the proof essentially due to Martin [15]. Let $M_{D, Q}^{K}(z)$ be the minimizing function belonging to $\mathscr{L}_{K}^{2}(D)$, then, using an orthonormal system $\phi_{D}(z)$ in $D$, we can set $M_{D, Q}^{K}(z)=A \phi_{D}(z)$, where $A \equiv\left(a_{i j}\right)=\int_{D} M_{D, Q}^{K}(\zeta) \phi_{D}^{*}(\zeta) \omega_{\zeta}$ denotes the Fourier coefficient $r \times \infty$ matrix to be determined. Noting that $\mathcal{L}_{M}^{K}, Q=A \mathcal{L}_{D}=A \Phi$, if we set

$$
I(A) \equiv\left(Q-M_{D, Q}^{K}, Q-M_{D, Q}^{K}\right)_{D}-\operatorname{Sp}\left\{(A \Phi-K) \Lambda+\Gamma^{*}\left(\Phi^{*} A^{*}-K^{*}\right)\right\},
$$

where $\Lambda=\left(\lambda_{i j}\right)$ and $\Gamma=\left(y_{i j}\right)(i=1, \ldots, p ; j=1, \ldots, r$ and $p$ denotes the number of the columns of $K$ ) are the Lagrangian multipliers, as necessary conditions we must have the Euler's conditions

$$
\partial I(A) / \partial a_{i j}=\bar{a}_{i j}-\bar{b}_{i j}-(\Phi,)_{i j}=0, \text { i.e., } A^{*}=B^{*}+\Phi \Lambda
$$

and

$$
\partial I(A) / \partial \bar{a}_{i j}=a_{i j}-b_{i j}-\left(\Gamma^{*} \Phi^{*}\right)_{i j}=0 \text {, i.e., } A=B+\Gamma^{*} \Phi^{*}
$$

where $i=1, \ldots, r ; j=1,2, \ldots$ and $(\Phi,)_{i j}$ denotes the $(i, j)$-element of $\Phi, 1$. Hence we have $\Phi \Lambda=\Phi \Gamma$. But since $\operatorname{det}\left(\Phi^{*} \Phi\right) \neq 0$ holds in a bounded domain, we obtain $\Lambda=\Gamma$. On the other hand, as we have $K=A \Phi=$ $\left(B+\Lambda^{*} \Phi^{*}\right) \Phi=B \Phi+\Lambda^{*}\left(\Phi^{*} \Phi\right)$, we get

$$
A=B+\Lambda^{*} \Phi^{*}=B+(K-B \Phi)\left(\Phi^{*} \Phi\right)^{-1} \Phi^{*} \text {. }
$$

Therefore, we must have (3.3) belonging to $\varrho_{K}^{2}(D)$.

In order to prove that $M_{D, Q}^{K}(z)$ is the minimizing function required, let us consider the class $\mathfrak{L}_{0}^{2}(D) \equiv\left\{g(z) \in \mathfrak{L}^{2}(D) \mid g(z)=C \phi_{D}(z), C \Phi=0\right\}$. If we set $F(z)=M_{D, Q}^{K}(z)+g(z)$ for each $g(z) \in \mathscr{L}_{0}^{2}(D)$, then it is easily shown that $F(z)$ is an arbitrary function belonging to $\mathfrak{L}_{K}^{2}(D)$. It follows from termby-term integrability (see [15]) that

$$
\begin{aligned}
& \int_{D}\left\{Q(\zeta, \bar{\zeta})-M_{D, Q^{(\zeta)}}^{K}\right\} g^{*}(\zeta) \omega_{\zeta} \\
& \quad=\int_{D} Q(\zeta, \bar{\zeta}) \phi_{D}^{*}(\zeta) \omega_{\zeta} C^{*}-A \int_{D} \phi_{D}(\zeta) \phi_{D}^{*}(\zeta) \omega_{\zeta} C^{*} \\
& \quad=B C^{*}-A C^{*}=B C^{*}-\left(B+\Lambda^{*} \Phi^{*}\right) C^{*}=-\Lambda^{*}(C \Phi)^{*}=0
\end{aligned}
$$

where $\int_{D} \phi_{D}(\zeta) \phi_{D}^{*}(\zeta) \omega_{\zeta}=E_{\infty}$. Hence we obtain 


$$
\begin{aligned}
(Q-F, Q-F)_{D}=\left(Q-M_{D, Q}^{K}, Q-M_{D, Q}^{K}\right)_{D}+(g, g)_{D} \\
\quad-\quad \operatorname{ReSp} \int_{D}\left(Q-M_{D, Q}^{K}\right) g^{*} \omega_{\zeta} \\
=\left(Q-M_{D, Q}^{K}, Q-M_{D, Q}^{K}\right)_{D}+(g, g)_{D}>\left(Q-M_{D, Q}^{K}, Q-M_{D, Q}^{K}\right)_{D}
\end{aligned}
$$

for any $g(z) \not 0$. This completes the proof.

Remark 3.1. In Theorem 3.1 it is easily verified that the minimizing function without an additional condition (3.2) is given by

$$
M_{D, Q}(z)=B \phi_{D}(z)=\int_{D} Q(\zeta, \bar{\zeta}) k_{D}(z, \bar{\zeta}) \omega_{\zeta}, \quad k_{D}(z, \bar{\zeta}) \equiv \phi_{D}^{*}(\zeta) \phi_{D}(z)
$$

where $k_{D}(z, \bar{\zeta})$ denotes the Bergman kernel function [15].

In the case that $Q(z, \bar{z}) \equiv 0$ in $D$, the minimizing function $M_{D}^{K}(z) \equiv$ $M_{D, Q=0}^{K}(z)$ and the minimum value $\lambda_{D}^{K} \equiv \lambda_{D, Q=0}^{K}$ are expressed in terms of the kernel function of $D$ and its derivatives [3], [20].

Let $\mathscr{L}_{(m)} \equiv\left(\mathscr{C}_{1}, \ldots, \mathfrak{L}_{m}\right)$ be an element of $B L(D)$ and $\mathscr{L}_{(m), t}$ and $\mathscr{L}_{k, t}$ be the bounded linear functionals $\mathscr{L}_{(m)}$ and $\mathscr{L}_{k}$ evaluated at a point $t \in D . \mathscr{L}_{K(m)}^{2}(D)$ and $\mathscr{\complement}_{K(m), t}^{2}(D)$ denote the subclasses of $\mathscr{\complement}^{2}(D)$ such that $\left\{f(z) \in \mathscr{L}^{2}(D) \mid \mathcal{Q}_{(m)} f=K(m) \equiv\left(A_{1}, \ldots, A_{m}\right)\right\}$ and $\left\{f(z) \in \mathscr{L}^{2}(D) \mid \mathscr{L}_{(m), t} f=K(m)\right\}$, respectively. Here $\mathfrak{L}_{k, t} f$ denotes, say, any one of $f(t), D_{z}^{k} f(t),\left(D_{z}^{k} f(t)\right) u^{k}$, $\int_{0}^{t} f(z) d z$ and $\int_{D} f(z) \omega_{z}$ and so on.

Theorem 3.1 gives the generalizations of (i) [15], (ii) [15, (5.5)], (iii) [3], [20] and (iv) [19] under the additional conditions

(i) $Q(z, \bar{z}) \in L^{2}(D), \mathscr{\complement}_{(m), t} f=\cdot K(m), t \in D$,

(ii) $Q(z, \bar{z}) \in L^{2}(D), \mathscr{\complement}_{(m)}^{f} f \equiv\left(\mathscr{\complement}_{1, t_{1}}, \ldots, \mathscr{L}_{m, t_{m}}\right) f \equiv\left(f\left(t_{1}\right), \ldots, f\left(t_{m}\right)\right)$ $=K(m), t_{k} \in D(\dot{k}=1, \ldots, m)$,

(iii) $)^{\prime} Q(z, \bar{z}) \equiv 0, \mathfrak{\complement}_{(m), t} f \equiv\left(\mathscr{\complement}_{1, t}, \ldots, \mathfrak{L}_{m, t}\right) f=K(m)$, where $\mathfrak{L}_{k, t} f \equiv$ $\left(D_{z}^{k} f(t)\right) u_{k}\left(u_{k}\right.$ denotes a constant ${ }_{n} H_{k} \times i_{k}$ matrix $(k=1, \ldots, m)$ and $i_{k}$ denotes an arbitrary integer belonging to $\left\{1,2, \ldots,{ }_{n} H_{k}\right\}\left({ }_{n} H_{k}\right.$ : repeated combination), and

$$
\text { (iv) } Q(z, \bar{z}) \equiv 0, \mathfrak{L}_{(2), t} f \equiv\left(\mathfrak{Q}_{1, t}, \mathfrak{Q}_{2, t}\right) f \equiv\left(f(t), \int_{D} f(\zeta) \omega_{\zeta}\right)=K(2) \text {, re- }
$$
spectively.

In the following we shall use the abbreviated notations $f_{i j}(z, \bar{x})$ and $f_{[i j]}(z, \bar{x})$ instead of $\left(D_{z}^{*}\right)^{i}\left(D_{z}\right)^{j} f(z, \bar{x})$ and $\left[D_{z}^{*}\right]^{i}\left[D_{z}\right]^{j} f(z, \bar{x})$, respectively. In particular, $f_{00}(z, \bar{x})$ denotes $f(z, \bar{x})$ and $f_{i j}(a, \bar{b})$ means $\left.f_{i j}(z, \bar{x})\right|_{z=a, x=b^{*}}$

In a bounded domain $D$, the Bergman kernel function $k_{D}(z, \bar{z})$ is positive and relatively invariant under $B H(D)$ and $\log k_{D}(z, \bar{z})$ defines a strongly 
plurisubharmonic function. Therefore, an absolutely invariant Kähler metric under $B H(D)$, which is called the Bergman metric, is defined as

$$
d s_{D}^{2} \equiv d z^{*} T_{D}(z, \bar{z}) d z,
$$

where the fundamental tensor

$$
\begin{aligned}
T_{D}(z, \bar{t}) & \equiv D_{t}^{*} D_{z} \log k_{D}(z, \bar{t}) \\
& =\left\{k(z, \bar{t}) \times k_{11}(z, \bar{t})-k_{10}(z, \bar{t}) \times k_{01}(z, \bar{t})\right\} / k^{2}(z, \bar{t})
\end{aligned}
$$

belongs to $H\left(D \times D^{*}\right)$ when $k(z, \bar{t}) \equiv k_{D}(z, \tau) \neq 0$ and has the relative invariancy under $B H(D)$, where $k_{i j}(z, \tau)$ denotes $k_{D, i j}(z, \tau)$, etc.

The following lemma is known [2], [3], [7].

Lemma 3.1. We consider the case that $Q(z, \bar{z}) \equiv 0$ in $D$.

(i) Under $\mathscr{L}_{(2), t} f \equiv\left(f(t), D_{z} f(t)\right)=K(2) \equiv\left(A_{1}, A_{2}\right)$ we have

$$
M_{D}^{K(2)}(z, t)=\left(A_{1}, A_{2}\right)\left(\begin{array}{ll}
k & k_{01} \\
k_{10} & k_{11}
\end{array}\right)^{-1}\left(\begin{array}{l}
k(z, \bar{t}) \\
k_{10}(z, \bar{t})
\end{array}\right),
$$

where

$$
\left(\begin{array}{ll}
k & k_{01} \\
k_{10} & k_{11}
\end{array}\right)^{-1}=\left(\begin{array}{cc}
1 / k+k_{01}(k T)^{-1} k_{10} / k^{2}, & -k_{01}(k T)^{-1 / k} \\
-(k T)^{-1} k_{10} / k, & (k T)^{-1}
\end{array}\right),
$$

$k_{i j} \equiv k_{D, i j}(t, \bar{t})$ and $T \equiv T_{D}(t, \bar{t})$.

In particular, under $K(2) \equiv\left(0, E_{n}\right)$ we have

$$
\lambda_{D}^{0 E_{n}}(t)=\mathrm{Sp}(k T)^{-1} \text {. }
$$

(ii) Under $\mathscr{L}_{(2), t} f \equiv\left(f(t), D_{z} f(t) u\right)=K(2) \equiv(0,1)$ we have

$$
\lambda_{D}^{01}(t) \equiv \lambda_{D}^{(2)}(u)=1 / k u^{*} T u .
$$

(iii) Under $\mathcal{L}_{(1), t} f \equiv(f(t))=K(1) \equiv(1)$ we have

$$
\lambda_{D}^{1}(t) \equiv \lambda_{D}^{(1)}=1 / k
$$

(iv) Under $\mathscr{L}_{(3), t} f \equiv\left(f(t), D_{z} f(t),\left[D_{z}\right]^{2} f(t)(u \times v)\right)=K(3) \equiv(0, \ldots, 0,1)$ we have 


$$
\lambda_{D}^{001}(t) \equiv \lambda_{D}^{(3)}(u, v)=\lambda_{D}^{(2)}(u) \lambda_{D}^{(2)}(v) / \lambda_{D}^{(1)}(u \times v)^{*} \Omega_{D}^{(2)}(t)(u \times v),
$$

where $\Omega_{D}^{(2)}(t)$ is defined in (4.5).

Remark 3.2. For a regular matrix $A \equiv\left(\begin{array}{ll}K & L \\ M\end{array}\right)$, if $K$ and $Z \equiv N-M K^{-1} L$ are regular, then we have

$$
A^{-1}=\left(\begin{array}{cc}
K^{-1}+X Z^{-1} Y, & -X Z^{-1} \\
-Z^{-1} Y, & Z^{-1}
\end{array}\right),
$$

where $X \equiv K^{-1} L$ and $Y \equiv M K^{-1}$.

4. New invariant Kähler metrics.

Definition 4.1. We define the two sorts of matrices:

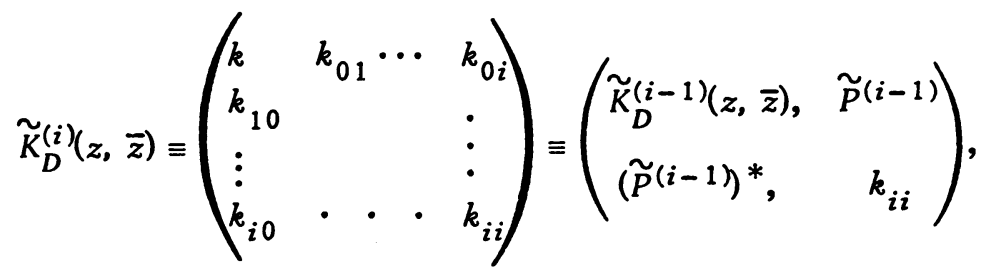

$i=0,1,2, \ldots$, and

(4.2) $K_{D}^{(i)}(z, \bar{z}) \equiv\left(\begin{array}{cc}\tilde{K}_{D}^{(i-1)}(z, \bar{z}), & P^{(i-1)} \\ -\left(P^{(i-1)}\right)^{*}, & \left(k_{i-1, i-1}\right)_{11}\end{array}\right), P^{(i-1)} \equiv\left(\begin{array}{c}\left(k_{0, i-1}\right)_{01} \\ \vdots \\ \left(k_{i-1, i-1}\right)_{01}\end{array}\right)$,

where $\left(k_{p q}\right)_{01}$ denotes $D_{z}\left\{\left(D_{z}^{*}\right)^{p} D_{z}^{q} k_{D}(z, \bar{z})\right\}$, etc., and $\widetilde{K}_{D}^{(i)}$ and $K_{D}^{(i)}$ are $s(i) \times s(i)$ and $\{s(i-1)+n t(i-1)\} \times\{s(i-1)+n t(i-1)\}$ matrices, respectively. Here $t(i)$ and $s(i)$ denote ${ }_{n} H_{i}$ and $\Sigma_{k=0}^{i} t(k)\left(=\left(\begin{array}{c}n+i \\ i\end{array}\right)\right)$, respectively.

Lemma 4.1. In a bounded domain $D$, we have

(4.3) $\operatorname{det} \tilde{K}_{D}^{(i)}(z, \bar{z})>0, \quad \operatorname{det} K_{D}^{(i)}(z, \bar{z}) \equiv 0 \quad(i \geq 2$ in the latter $)$,

$$
\tilde{\Omega}_{D}^{(i)}(z) \equiv\left\{k_{i i}-\left(\tilde{P}^{(i-1)}\right) *\left(\tilde{K}_{D}^{(i-1)}\right)-1 \tilde{P}^{(i-1)}\right\} / k>0
$$

and

$$
\Omega_{D}^{(i)}(z) \equiv\left\{\left(k_{i-1, i-1}\right)_{11}-\left(P^{(i-1)}\right) *\left(\tilde{K}_{D}^{(i-1)}\right)-1 P^{(i-1)}\right\} / k,
$$

$$
\left(u^{*} \times E_{t(i-1)}\right) \Omega_{D}^{(i)}(z)\left(u \times E_{t(i-1)}\right)>0
$$


for $i \geq 0$, where $t(i-1)={ }_{n}{ }_{i-1}$.

Proof. det $\tilde{K}_{D}^{(0)}(z, \bar{z})=k_{D}(z, \bar{z})>0$ in $D$ is clear. Since $k_{D}^{-1}$ exists, then we have $\operatorname{det} K_{D}^{(1)} \equiv k_{D}^{n+1}(z, \bar{z}) \operatorname{det} T_{D}(z, \bar{z})>0$ in $D$.

Now, let us suppose that det $\tilde{K}_{D}^{(i-1)}(z, \bar{z})>0$ in $D$. Under the condition $\left(f(t), D_{z} f(t), \ldots,\left(D_{z}^{i} f(t)\right) v\right)=(0, \ldots, 0,1) \equiv K(i+1)$, where $v$ denotes any nonzero ${ }_{n}{ }_{i} \times 1$ vector, we obtain, from (3.4),

$$
\begin{aligned}
\tilde{\lambda}_{D}^{K(i+1)}(t) & =\operatorname{det} \tilde{K}_{D}^{(i-1)} / \operatorname{det}\left(\begin{array}{cc}
\tilde{K}_{D}^{(i-1)}, & \tilde{P}^{(i-1)} v \\
v^{*}\left(\tilde{P}^{(i-1)} *,\right. & v^{*} k_{i i} v
\end{array}\right) \\
& =1 / k v^{*} \tilde{\Omega}_{D}^{(i)}(t) v>0
\end{aligned}
$$

and hence $\tilde{\Omega}_{D}^{(i)}(z)$ is positive definite and also det $\tilde{\Omega}_{D}^{(i)}(z)>0$ follows. Therefore, we have, from (4.1) and (4.4),

$$
\operatorname{det} \widetilde{K}_{D}^{(i)}(z, \bar{z})=k \operatorname{det} \widetilde{K}_{D}^{(i-1)}(z, \bar{z}) \operatorname{det} \widetilde{\Omega}_{D}^{(i)}(z)>0 .
$$

Under the condition $\left(f(t), D_{z} f(t), \ldots, D_{z}^{i-1} f(t),\left.D_{z} D_{z}^{i-1} f(z)\right|_{z=t}(u \times v)\right)$ $=(0, \ldots, 0,1) \equiv K(i+1)$, where $u$ and $v$ are $n \times 1$ and ${ }_{n}{ }_{i-1} \times 1$ constant vector respectively, we have, by the same procedure as above,

$$
(u \times v) * \Omega_{D}^{(i)}(t)(u \times v)=v^{*}\left(u^{*} \times E_{t(i-1)}\right) \Omega_{D}^{(i)}(t)\left(u \times E_{t(i-1)}\right) v>0,
$$

which shows (4.5).

Lemma 4.2. In a bounded domain $D$, we have

$$
\begin{aligned}
& \partial_{z}^{*} \partial_{z} \log \operatorname{det} \tilde{K}_{D}^{(i)}(z, \bar{z}) \\
& \quad=\operatorname{Sp}\left\{\left(\tilde{\Omega}_{D}^{(i)}\right)-1\left(d z^{*} \times E_{t(i)}\right) \Omega_{D}^{(i+1)}(z)\left(d z \times E_{t(i)}\right)\right\}>0 .
\end{aligned}
$$

Proof. Noting that $\tilde{\Omega}_{D}^{(i)}(z)$ and $\left(u^{*} \times E_{t(i)}\right) \Omega_{D}^{(i+1)}(z)\left(u \times E_{t(i)}\right)$ are positive definite from (4.4), we have by Lemma 2.1

$\partial_{z}^{*} \partial_{z} \log \operatorname{det} \tilde{K}_{D}^{(i)}$

$$
\begin{aligned}
& \left.=\operatorname{Sp}\left[\left(\tilde{K}_{D}^{(i)}\right)^{-1}\left(d z^{*} \times E_{s(i)}\right) \tilde{K}_{D, 11}^{(i)}-\tilde{K}_{D, 10}^{(i)}\left(\tilde{K}_{D}^{(i)}\right)-1 \tilde{K}_{D, 01}^{(i)}\right\}\left(d z \times E_{s(i)}\right)\right] \\
& =\operatorname{Sp}\left\{\left(\begin{array}{cc}
* & * \\
* & \left(k \tilde{\Omega}_{D}^{(i)}\right)-1
\end{array}\right)\left(\begin{array}{cc}
d z^{*} & 0 \\
0 & d z^{*} \times E_{t(i)}
\end{array}\right)\left(\begin{array}{cc}
0 & 0 \\
0 & k \Omega_{D}^{(i+1)}
\end{array}\right) \cdot\left(\begin{array}{cc}
d z & 0 \\
0 & d z \times E_{t(i)}
\end{array}\right)\right\} \\
& =\operatorname{Sp}\left\{\left(\tilde{\Omega}_{D}^{(i)}\right)^{-1}\left(d z^{*} \times E_{t(i)}\right) \Omega_{D}^{(i+1)}\left(d z \times E_{t(i)}\right)\right\}>0,
\end{aligned}
$$


where $s(i)=1+{ }_{n} H_{1}+\cdots+{ }_{n} H_{i}=\left(\begin{array}{c}n+i \\ i\end{array}\right)$ and $t(i)={ }_{n}{ }_{i}$, since $\mathrm{Sp}\left(H_{1} H_{2}\right)>0$ follows when $H_{1}$ and $H_{2}$ are positive definite Hermitian matrices.

Definition 4.2. Such an ${ }_{n}{ }_{i} \times{ }_{n}{ }_{j}$ matrix $O(A)$ that

$$
\left([v]^{i}\right)^{T} A[u]^{j}=\left(v^{i}\right)^{T} \sigma(A) u^{j}
$$

holds for arbitrary nonzero vectors $u \equiv\left(u_{1}, \ldots, u_{n}\right)^{T}$ and $v \equiv\left(v_{1}, \ldots, v_{n}\right)^{T}$ is called the $\sigma$-contraction of an $n^{i} \times n^{i}$ matrix $A$.

Further, for a linear transformation $v=A u$ we define another contraction $\delta[A]^{k}$ of $[A]^{k}$ as follows: $v^{k}=\left(\delta[A]^{k}\right) u^{k}$, where $u, v$ and $A$ denote $n \times 1, m \times 1$ vectors and an $m \times n$ matrix, respectively.

Lemma 4.3. Let $g(z, \bar{z})$ and $w(z)$ be a scalar function and a biholomorphic mapping in $D$, then we have

$$
\delta[A B]^{k}=\left(\delta[A]^{k}\right)\left(\delta[B]^{k}\right),
$$

in particular, $\delta[u]^{k}=u^{k}$ and $\delta[A u]^{k}=\left(\delta[A]^{k}\right) u^{k}$, and further we have

$$
\begin{aligned}
\sigma\left(g_{[i j}\right) & =g_{i j}, \\
\left.\sigma\left(\left[D_{z} w\right]^{i}\right)^{*} g_{[i j]}\left[D_{z} w\right]^{j}\right\} & =\delta\left(\left[D_{z} w\right]^{i}\right)^{*} g_{i j} \delta\left[D_{z} w\right]^{j} .
\end{aligned}
$$

For an $n \times n$ matrix $C$ and a natural number $k$ we have

$$
\operatorname{det} \delta[C]^{k}=(\operatorname{det} C)^{s(k-1)}, \quad s(k-1)=\left(\begin{array}{c}
n+k-1 \\
k-1
\end{array}\right) .
$$

Proof. (4.8) and (4.9) are evident from Definition 4.2.

By the triangulation of $C$ we have $C=P S P^{-1}$, where $P$ and $S$ denote $n \times n$ regular and $n \times n$ triangular matrices, respectively. Since $[C]^{k}=$ $[P]^{k}[S]^{k}\left[P^{-1}\right]^{k}$ and $\delta\left[P^{-1}\right]^{k}=\delta\left([P]^{k}\right)^{-1}=\left(\delta[P]^{k}\right)^{-1}$ hold, then we obtain $\operatorname{det}\left(\delta[C]^{k}-\lambda E_{n}\right)=\operatorname{det}\left(\delta[S]^{k}-\lambda E_{n}\right)$, which derives (4.10). $s(k-1)$ is obtained from $t(k) \times k / n={ }_{n} H_{k} k / n={ }_{n+1} H_{k-1}=\left(\begin{array}{c}n+k-1 \\ k-1\end{array}\right)$.

Lemma 4.4. Under $w(z) \in B H(D)$ we have the relative invariances:

$$
\operatorname{det} \tilde{K}_{D}^{(i)}(z, \bar{z})=\operatorname{det} \tilde{K}_{\Delta}^{(i)}(w, \bar{w})\left|J_{w}(z)\right|^{2 N(i)}, \quad i \geq 0,
$$

and have the absolute invariants:

$$
I_{D}^{(i)}(z) \equiv \operatorname{det} \tilde{K}_{D}^{(i)}(z, \bar{z}) /\left(k_{D}(z, \bar{z})\right)^{N(i)}, \quad i \geq 0,
$$

where $\Delta=w(D)$ and $N(i) \equiv\left(\begin{array}{c}n+i+1 \\ i\end{array}\right)$. 
In particular, for $i=1$ we have a known absolute invariant:

$$
I_{D}^{(1)}(z) \equiv \operatorname{det} T_{D}(z, \bar{z}) / k_{D}(z, \bar{z}) \quad \text { [3]. }
$$

Proof. The Bergman kernel function $k_{D}(z, \bar{z})$ has the relative invariancy:

$$
k_{D}(z, \bar{z})=\bar{J} k_{\Delta}(w, \bar{w}) J \text { for } w(z) \in B H(D),
$$

where $J \equiv J_{w}(z)=\operatorname{det}\left(D_{z} w\right)$. Let us set $k_{D}(z, \bar{z}) \equiv k_{D}$ and $k_{\Delta}(w, \bar{w}) \equiv k_{\Delta}$; then we have

Since

$$
\left[D_{z}^{*}\right]^{p}\left[D_{z}\right]^{q} k_{\Delta}=\left(\left[D_{z} w\right]^{p}\right) *\left(\left[D_{w}^{*}\right]^{p}\left[D_{w}\right]^{q} k_{\Delta}\right)\left[D_{z} w\right]^{q}
$$

$$
\begin{aligned}
& {\left[D_{z}^{*}\right]^{p}\left[D_{z}\right]^{q} k_{D} }=\left[D_{z}^{*}\right]^{p}\left[D_{z}\right]^{q}\left(\bar{j} k_{\Delta} J\right) \\
&=\sum_{j=0}^{p} \sum_{i=0}^{q}\left({ }_{p} C_{j} \times{ }_{q} C_{i}\right)\left\{\left(\left[D_{z} w\right]^{p-j}\right)^{*} \times \bar{J}_{[j 0]}\right\} \\
&\left.\left.\cdot k_{\Delta,[p-j, q-i}\right]^{\left[\dot{D}_{z} w\right]^{q-i}} \times J_{[0 i]}\right\},
\end{aligned}
$$

using the elementary theorems with respect to the determinant and the contraction

$$
\sigma\left(\left[D_{z}^{*}\right]^{p}\left[D_{z}\right]^{q} k_{\Delta}\right)=\delta\left(\left[D_{z} w\right]^{p}\right)^{*} k_{\Delta, p q} \delta\left[D_{z} w\right]^{q},
$$

we have, by (4.10),

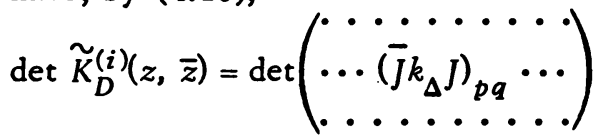

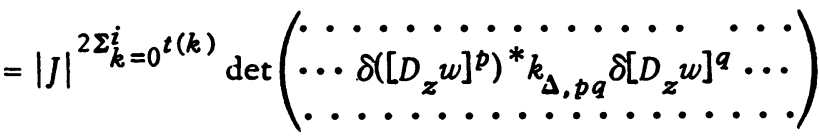

$$
\begin{aligned}
& =|J|^{2 \Sigma_{k=0}^{i} t(k)}\left|\prod_{q=1}^{i} \operatorname{det} \delta\left[D_{z} w\right]^{q}\right|^{2} \operatorname{det} \tilde{K}_{\Delta}^{(i)}(w, \bar{w}) \\
& =|J|^{2\left(\Sigma_{k}^{i}=0^{t(k)+\Sigma_{k}^{i}=1} s(k)\right)} \operatorname{det} \tilde{K}_{\Delta}^{(i)}(w, \bar{w}),
\end{aligned}
$$

where $t(k)={ }_{n} H_{k}$ and $s(k)=\left(\begin{array}{c}n+k \\ k\end{array}\right)$. Since

$$
\sum_{k=0}^{i} t(k)+\sum_{k=0}^{i-1} s(k)=\left(\begin{array}{c}
n+i \\
i
\end{array}\right)+\left(\begin{array}{c}
n+i \\
i-1
\end{array}\right)=\left(\begin{array}{c}
n+i+1 \\
i
\end{array}\right) \equiv N(i),
$$

we have (4.11) and thus (4.12). 
Theorem 4.1. In a bounded domain $D$

$$
\left(d s_{D}^{(i)}\right)^{2} \equiv \partial_{z}^{*} \partial_{z} \log \operatorname{det} \widetilde{K}_{D}^{(i)}(z, \bar{z}), \quad i=0,1,2, \cdots,
$$

define the new invariant Kähler metrics under $B H(D)$ (see (4.7)).

Proof. The positivity of each $\left(d s_{D}^{(i)}\right)^{2}$ is given by Lemma 4.2.

From Lemma 4.4 we can obtain the invariancy of $\left(d s_{D}^{(i)}\right)^{2}$ under $B H(D)$, since we have

$$
\log \operatorname{det} \widetilde{K}_{D}^{(i)}(z, \bar{z})=\log \operatorname{det} \widetilde{K}_{\Delta}^{(i)}(w, \bar{w})+\psi(z)+\overline{\psi(z)},
$$

where $\psi(z)$ denotes the scalar analytic function $N(i) \log J_{w}(z)$, and $\partial_{z} f(z)$ $=\left(D_{w} f(z(w))\left(D_{z} w\right)\right) d z=\left(D_{w} F(w)\right) d w$ holds for a holomorphic function $f(z)=$ $f(z(w)) \equiv F(w)$ under $w(z) \in B H(D)$.

Remark 4.1. $\left(d s_{D}^{(0)}\right)^{2}$ and $\left(d s_{D}^{(1)}\right)^{2}$ coincide with the Bergman metric [3] and the Fuks metric [8], respectively.

Corollary 4.1. In a bounded domain $D$, we have

$$
-\left(R_{\bar{a} \beta}\right)+(n+1) T_{D}=D_{z}^{*} D_{z} \log \operatorname{det} K_{D}^{(1)} \quad(c f .[13])
$$

and for any nonzero vector $u$

$$
u^{*}\left(D_{z}^{*} D_{z} \log \operatorname{det} K_{D}^{(1)}\right) u=\operatorname{Sp}\left\{T_{D}^{-1}\left(u^{*} \times E_{n}\right) \Omega_{D}^{(2)}(z)\left(u \times E_{n}\right)\right\}>0
$$

where, for the Hermitian curvature tensor $\left(-R_{\bar{\alpha} \beta \bar{\gamma}} \delta\right.$,

$$
\left(R_{\bar{a} \beta}\right) \equiv\left(\sum_{\gamma \delta} T^{\bar{\gamma} \delta}\left(-R_{\bar{\alpha} \beta \bar{\gamma} \delta}\right)\right)=-D_{z}^{*} D_{z} \log \operatorname{det} T_{D}, \quad\left(T^{\bar{\gamma} \delta}\right)=T_{D}^{-1}
$$

[13] denotes the Ricci tensor with respect to the Bergman metric.

Proof. By Lemma 4.2 we have

$$
\begin{aligned}
\partial_{z}^{*} \partial_{z} \log \operatorname{det} K_{D}^{(1)} & =\partial_{z}^{*} \partial_{z} \log \left(k^{n+1} \operatorname{det} T\right)=d z^{*}\left(-\left(R_{\bar{a} \beta}\right)+(n+1) T\right) \\
& =\operatorname{Sp}\left\{\left(\widetilde{\Omega}_{D}^{(1)}(z)\right)^{-1}\left(d z^{*} \times E_{n}\right) \Omega_{D}^{(2)}(z)\left(d z \times E_{n}\right)\right\} \\
& =\operatorname{Sp}\left\{T^{-1}\left(d z^{*} \times E_{n}\right) \Omega_{D}^{(2)}(z)\left(d z \times E_{n}\right)\right\}>0
\end{aligned}
$$

since $\widetilde{K}_{D}^{(1)} \equiv K_{D}^{(1)}$ holds, where $k \equiv k_{D}(z, \bar{z})$ and $T \equiv T_{D}(z, \bar{z})$.

Corollary 4.2. In a bounded domain $D$, let us set 


$$
J_{D,(p, q)}(z, \bar{z}) \equiv \operatorname{det}\left(k_{D}^{p}(z, \bar{z}) \times T_{D}^{q}(z, \bar{z})\right),
$$

which is relatively invariant under $B H(D)$ for arbitrary real number $p$ and integer $q$; then

$$
d s_{D,(p, q)}^{2} \equiv \partial_{z}^{*} \partial_{z} \log J_{D,(p, q)}(z, \bar{z}) \quad\left(\equiv d z^{*} T_{D,(p, q)}(z, \bar{z}) d z\right)
$$

defines an invariant Käbler metric under $B H(D)$ for each $(p, q)$ such that $n p-(n+1) q \geq 0(n=\operatorname{dim} D)$. Here $k_{D}^{p}(z, \bar{z})$ takes values of the real positive branch.

$$
\begin{aligned}
& \text { Proof. Since } J_{D_{,}(p, q)}(z, \bar{z})=k_{D}^{p n}(z, \bar{z})\left(\operatorname{det} T_{D}(z, \bar{z})\right)^{q} \text {, then } \\
& \partial^{2} \log J_{D,(p, q)} / \partial z^{*} \partial z=p n T_{D}-q\left(R_{\bar{\alpha} \beta}\right)>p n T_{D}-q(n+1) T_{D} \geq 0
\end{aligned}
$$

follows from (4.18) and (4.19). The invariancy of $d s_{D,(p, q)}^{2}$ follows from the relative invariancies of $k_{D}$ and $T_{D}$. We can obtain the relative invariance $J_{D,(p, q)}(z, \bar{z})=J_{\Delta,(p, q)}(w, \bar{w})\left|J_{w}(z)\right|^{2(p n+q)}$ for $w(z) \in B H(D)$ and $\Delta=w(D)$, where $\left|J_{w}(z)\right|^{2(p n+q)}$ takes values of the real poșitive branch.

Remark 4.2. The particular case of $(p, q)=((n+1) / n, 1)(n=\operatorname{din} D)$ was treated by Fuks [8] and $d s_{D,((n+1) / n, 1)}^{2}$ coincides with $\left(d s_{D}^{(2)}\right)^{2}$. For $(p, q)=(2,1), d s_{D,(2,1)}^{2}$ coincides with the Kato metric [11], which is valid for arbitrary $n(n=\operatorname{dim} D)$ and for $(p, q)=(1,0), d s_{n,(1,0)}^{2}$ denotes the Bergiman metric.

Under the restriction $q=1$ and $p \geq(n+1) / n$, (i) the possible minimum value of $p$ for each $n(n=\operatorname{dim} D)$ equals $(n+1) / n$, which is the case of Fuks, and (ii) the possible maximum value of $p$ for all $n(n=\operatorname{dim} D \geq 1)$ equals 2, which is the case of Kato.

If $D$ is a bounded homogeneous domain, $d s_{D,(p, q)}^{2}$ is essentially equivalent to the Bergman metric for $p n+q>0$.

Corollary 4.3. In a bounded domain $D$, we have

$$
\begin{gathered}
\Omega_{D}^{(2)}(z)=K_{[22,00]}-K_{[21,00]^{T}} K_{[21,00]}^{*}, \\
\left(u^{*} \times E_{n}\right) \Omega_{D}^{(2)}(z)\left(u \times E_{n}\right)>0 \quad \text { (positive definite) in } D
\end{gathered}
$$

and further

$$
\tilde{\Omega}_{D}^{(2)}(z)=K_{22,00}-K_{21,00} T^{-1} K_{21,00}^{*}>0 \text { in } D .
$$


Here, $K_{[i j, s t]}=\left(_{[i j]} \times k_{[s t]}-k_{[i t]} \times k_{[s j]}\right) / k^{2}$,

$$
K_{i j, s t}=\left(k_{i j} \times k_{s t}-k_{i t} \times k_{s j}\right) / k^{2},
$$

$T \equiv T_{D}(z, \bar{z})$ and $k \equiv k_{D}(z, \bar{z})$, and $u$ and $v$ denote nonzero $n \times 1$ vectors.

Proof. From (4.5) we have

$$
\Omega_{D}^{(2)}(z)=\left\{k_{[22]}-\left(P^{(1)}\right) *\left(\tilde{K}_{D}^{(1)}\right)-1 P^{(1)}\right\} / k^{2} .
$$

Noting (3.9), we have (4.24) by straight calculations.

It is known [3] that the llermitian curvature tensor $\left(-R_{\bar{\alpha} \beta \bar{\gamma} \delta}\right)$ of the first kind with respect to the Bergman metric $d s^{2}=d z^{*} T_{D}(z, \bar{z}) d z$ of $D$ is given by

$$
\begin{aligned}
\left(-R_{\bar{a} \beta \bar{\gamma} \delta}\right) & =-T_{2, D}(z, \bar{z}) \\
& \equiv-\left(T_{11}-T_{10} T^{-1} T_{01}\right)=-\left(E_{n} \times T\right) D_{z}^{*}\left(T^{-1} D_{z} T\right),
\end{aligned}
$$

where $T \equiv T_{D}(z, \bar{z})$ and $T^{-1} D_{z} T$ denotes the matrix of the Christoffel symbols.

Theorem 4.2. The Hermitian curvature tensor with respect to the Bergman metric has the following expression:

(4.28) $-T_{2, D}(z, \bar{z})=\left(T_{D}(z, \bar{z}) \times T_{D}(z, \bar{z})\right)\left(E_{n} \times E_{n}+\widetilde{E}_{n n}\right)-\Omega_{D}^{(2)}(z)$

(cf. [13]).

$$
\Omega_{D}^{(2)}(z) \text { is a relative invariant under } B H(D) \text {. }
$$

Proof. Noting that $k_{[i j]}^{*}=k_{[j i]}, k_{[i 0]} \times k_{[0 j]}=k_{[0 j]} \times k_{[i 0]}, T_{01}^{*}=T_{10}$ and $D_{z}^{*} H=\left(D_{z} H\right)^{*}$ for an Hermite matrix $H(z, \bar{z})$, we have, by differentiating both sides of $k^{2} \times T=k \times k_{11}-k_{10} \times k_{01}$ with respect to $z$ and $z^{*}$,

$$
\begin{aligned}
k^{2} \times\left(D_{z}^{*} D_{z}\left(k^{2} \times T\right)\right) & -\left(D_{z}^{*}\left(k^{2} \times T\right)\right) T^{-1}\left(D_{z}\left(k^{2} \times T\right)\right)=k^{4} \times\left(T_{2, D}+2 T \times T\right) \\
= & k^{2} \times\left(k \times k_{[22]}-k_{[20]} \times k_{[02]}\right) \\
& -\left(k \times k_{[21]}-k_{[20]} \times k_{01}\right) T^{-1}\left(k \times k_{[12]}-k_{10} \times k_{[02]}\right) \\
& +k^{2} \times k_{11} \times k_{11}\left(E_{n} \times E_{n}-\tilde{E}_{n n}\right) \\
& -\left(k_{10} \times k_{11}-k_{11} \times k_{10}\right) T^{-1}\left(k_{01} \times k_{11}-k_{11} \times k_{01}\right)
\end{aligned}
$$

since $k_{01} \times k_{11}-k_{11} \times k_{01}=k \times\left(k_{01} \times T-T \times k_{01}\right)$. Noting that 


$$
\begin{gathered}
\left(k_{10} \times T\right) \times k_{01}=\left\{T \times\left(k_{10} \times k_{01}\right)\right\} \tilde{E}_{n n}, \\
\left(k_{10} \times k_{01}\right) \times\left(k_{10} \times k_{01}\right)=\left\{\left(k_{10} \times k_{01}\right) \times\left(k_{10} \times k_{01}\right)\right\} \tilde{E}_{n n}
\end{gathered}
$$

and

$$
k_{11} / k=T+k_{10} \times k_{01} / k^{2}
$$

we obtain

$$
\begin{aligned}
\left(k_{11} \times k_{11}\right)\left(E_{n} \times E_{n}-\widetilde{E}_{n n}\right) / k^{2} & \\
& \quad-\left(k_{10} \times k_{11}-k_{11} \times k_{10}\right) T^{-1}\left(k_{01} \times k_{11}-k_{11} \times k_{01}\right) / k^{4} \\
= & (T \times T)\left(E_{n} \times E_{n}-\widetilde{E}_{n n}\right) .
\end{aligned}
$$

Thus we get (4.28).

Since $T \times T$ and $T_{2, D}$ are relatively invariant under $B H(D)$ [10], [13], [14] and $\left[D_{z} w\right]^{2} \widetilde{E}_{n n}=\widetilde{E}_{n n}\left[D_{z} w\right]^{2}$ holds, then it follows from (4.28) that $\Omega_{D}^{(2)}(z)$ is relatively invariant under $B H(\dot{D})$.

Theorem 4.3. For each $i(i=0,1,2, \ldots)$ the mapping

$$
w_{D}^{(i)}(z) \equiv T_{D}^{(i)}(t, \bar{t}) \int_{t}^{z} T_{D}^{(i)}(z, \bar{t}) d z+t, \quad t \in D,
$$

defines the ith representative function, i.e., any domain $\Delta$ in the equivalent class $F \equiv\left\{f(D) \mid f(z) \in B H(D), f(t)=t, D_{z} f(t)=E_{n}\right\}$ is mapped onto the (unique) ith representative domain with center at $t$ by the function $w=w_{\Delta}^{(i)}(z)$, where $T_{D}^{(i)}(z, \bar{z})$ denotes the fundamental tensor $D_{z}^{*} D_{z} \log \operatorname{det} \tilde{K}_{D}^{(i)}(z, \bar{z})$ for the ith metric (4.18).

$A$ bounded domain $D$ is an ith representative domain with center at $t$ if and only if

$$
T_{D}^{(i)}(z, \bar{t})=T_{D}^{(i)}(t, \bar{t}) \quad \text { in } D
$$

holds (see [17]).

Proof. Since $T_{D}^{(i)}(z, \bar{t})$ is relatively invariant under $B H(D)$, then we have $w=w_{D}^{(i)}(z)=w_{\Delta}^{(i)}(\zeta)$ under any $\zeta=\zeta(z) \in F$. The latter half of the theorem is easily obtained by $w_{D}^{(i)}(z) \equiv z$ in $D$.

5. Curvatures and estimations. For the general sectional curvature 
$R_{D}(z ; u, v, u, v)$ (which is the expression in differential geometry) and a complex structure $J$, the holomorphic bisectional curvature with respect to the Bergman metric is defined as $R_{D}(z ; u, J u, v, J v)$ (S. Kobayashi). After some direct calculations we can show that $R_{D}(z ; u, J u, v, J v)$ coincides with the unitary curvature $R_{D}(z ; u, v)$ due to Hua [10] (see (4.27)). Now, we shall give the matrix expressions of the holomorphic bisectional curvature $R_{D}(z ; u, v)$ (of course, $R_{D}(z ; u, u)$ coincides with the holomorphic sectional curvature $\left.R_{D}(z ; u)\right)$, the Ricci curvature

$$
C_{D}(z ; u) \equiv u^{*}\left(R_{\dot{\bar{a} \beta}}\right) u / u^{*} T_{D^{u}}
$$

and the Ricci scalar curvature

$$
S_{D}(z) \equiv \operatorname{Sp}_{p}\left\{T_{D}^{-1}\left(R_{\bar{\alpha} \beta}\right)\right\}=\sum_{\bar{\alpha} \beta \bar{\gamma} \delta} T^{\bar{\alpha} \beta} T^{\bar{\gamma} \delta}\left(-R_{\bar{\alpha} \beta \bar{\gamma} \delta}\right)
$$

in terms of $T \equiv T_{D}(z, \bar{z})$ (Bergman metric tensor) and $\dot{T}_{2, D} \equiv T_{2, D}(z, \bar{z})$ (see (4.27) and (4.28)).

Lemma 5.1. For a bounded domain $D$ in $C^{n}$ and contravariant section vectors $u$ and $v$, we have

and

$$
\begin{gathered}
R_{D}(z ; u, v)=-(u \times v)^{*} T_{2, D}(u \times v) / u^{*} T u v^{*} T v, \\
C_{D}(z ; u)=-\operatorname{Sp}\left\{T^{-1}\left(u^{*} \times E_{n}\right) T_{2, D}\left(u \times E_{n}\right)\right\} / u^{*} T u
\end{gathered}
$$

which are absolute invariants under $B H(D)$.

Proof. Using the formula (2.5) and (4.19), we obtain

$$
\begin{aligned}
C_{D}(z ; u) & =-u^{*}\left(D_{z}^{*} D_{z} \log \operatorname{det} T\right) u / u^{*} T u \\
& =-\operatorname{Sp}\left\{T^{-1}\left(u^{*} \times E_{n}\right) T_{2, D}\left(u \times E_{n}\right)\right\} / u^{*} T u .
\end{aligned}
$$

$S_{D}(z) \equiv \Sigma T^{\bar{a} \beta} T^{\bar{\gamma} \delta}\left(-R_{\bar{a} \beta \bar{\gamma} \delta}\right)=-S_{\mathrm{p}}\left\{\left(T^{-1} \times T^{-1}\right) T_{2, D}\right\}$ is evident.

The biholomorphic invariancies of (5.1), (5.2) and (5.3) are easily obtained by the relative invariancies of $T$ and $T_{2, n}$ under $B H(D)$ [14].

For an $n \times n$ matrix $B=\left(b_{i j}\right)$ and $n \times 1$ vectors

$$
M_{i} \equiv(0, \ldots, 0,1,0, \ldots, 0)^{T},
$$


where 1 occurs in the $i$ th position $(i=1, \ldots, n)$, we have

$$
M_{j}^{T} B M_{i}=b_{j i}, \quad \sum_{i=1}^{n} M_{i}^{T} B M_{i}=\mathrm{Sp}(B) .
$$

Lemma 5.2. Let $v_{i}$ be the mutually orthogonal sections $T^{-1 / 2} M_{i}$ $(i=1, \ldots, n)$ such that $v_{j}^{*} T v_{i}=M_{j}^{T} M_{i}=\delta_{i j}$; then we have

$$
C_{D}(z ; u)=\sum_{i=1}^{n} R_{D}\left(z ; u, v_{i}\right)
$$

and

$$
S_{D}(z)=\sum_{j=1}^{n} C_{D}\left(z ; v_{j}\right)=\sum_{i, j=1}^{n} R_{D}\left(z ; v_{j}, v_{i}\right) .
$$

Proof. From (5.1) for $v=v_{i}$, noting $v_{i}^{*} T v_{i}=1$, we have

$$
R_{D}\left(z ; u, v_{i}\right)=-M_{i}^{T} T^{-1 / 2}\left(u^{*} \times E_{n}\right) T{ }_{2, D}\left(u \times E_{n}\right) T^{-1 / 2} M_{i} / u^{*} T u .
$$

By summation with respect to $i$ we obtain, from (5.2),

$$
\sum_{i=1}^{n} R_{D}\left(z ; u, v_{i}\right)=-\operatorname{Sp}\left\{T^{-1}\left(u \times E_{n}\right) T_{2, D}\left(u \times E_{n}\right)\right\} / u^{*} T u=C_{D}(z ; u) .
$$

By the same procedure, we have

$$
\begin{aligned}
\sum_{j=1}^{n} \sum_{i=1}^{n} R_{D}\left(z ; v_{j}, v_{i}\right) & =\sum_{j=1}^{n} C_{D}\left(z ; v_{j}\right) \\
& =-\operatorname{Sp}\left\{\left(T^{-1 / 2} \times T^{-1 / 2}\right) T_{2, D}\left(T^{-1 / 2} \times T^{-1 / 2}\right)\right\} \\
& =-\operatorname{Sp}\left\{\left(T^{-1} \times T^{-1}\right) T_{2, D}\right\}=S_{D}(z) .
\end{aligned}
$$

Theorem 5.1. Let $\lambda^{(1)}, \lambda^{(2)}(u)$ and $\lambda^{(3)}(u, v)$ be the minimum values in (3.12), (3.11) and (3.13) at $z$ in a bounded domain $D$, respectively, and $\epsilon \equiv \epsilon_{D}(z ; u, v)$ be $\left|u^{*} T v\right|^{2} / u^{*} T u v^{*} T v(0 \leq \epsilon \leq 1$ for $n \geq 2$ and $\epsilon=1$ for $n=1$ and $\left.\epsilon_{D}(z ; u, u)=1\right)$; then we have, for any sections $u$ and $v$,

$$
\begin{aligned}
R_{D}(z ; u, v) & =1+\epsilon-(u \times v) * \Omega(u \times v) / u^{*} T u v^{*} T v \\
& =1+\epsilon-\lambda^{(2)}(u) \lambda^{(2)}(v) / \lambda^{(1)} \lambda^{(3)}(u, v)<2 \quad \text { (cf. [2], [6], [19]), }
\end{aligned}
$$


and

$$
C_{D}(z ; u)=n+1-\operatorname{Sp}\left\{T^{-1}\left(u^{*} \times E_{n}\right) \Omega\left(u \times E_{n}\right)\right\} / u^{*} T u
$$

$$
=n+1-\lambda^{(2)}(u) \sum_{i=1}^{n}\left(\lambda^{(3)}\left(u, v_{i}\right)\right)^{-1}<n+1 \quad(c f .[4])
$$

$$
S_{D}(z)=n(n+1)-S_{p}\left\{\left(T^{-1} \times T^{-1}\right) \Omega\right\}
$$

$$
=n(n+1)-\lambda^{(1)} \sum_{i, j=1}^{n}\left(\lambda^{(3)}\left(v_{i}, v_{j}\right)\right)^{-1}<n(n+1)
$$

where $\Omega \equiv \Omega_{D}^{(2)}(z)$ and $v_{i} \equiv T^{-1 / 2} M_{i}(i=1, \ldots, n)$ are given in (4.4) and Lemma 5.2, respectively.

Proof. By (5.1), (4.28) and Lemma 3.1 we have (5.7).

Since it follows from (3.11) that $\lambda^{(2)}\left(v_{i}\right)=\lambda^{(1)}$, then we have

$$
\begin{aligned}
C_{D}(z ; u) & =\sum R_{D}\left(z ; u, v_{i}\right) \\
& =n+\left[\sum\left|u^{*} T^{1 / 2} M_{i}\right|^{2}-S_{\mathrm{p}}\left\{T^{-1}\left(u^{*} \times E_{n}\right) \Omega\left(u \times E_{n}\right)\right\}\right] / u^{*} T u \\
& =n+1-\operatorname{Sp}_{p}\left\{T^{-1}\left(u^{*} \times E_{n}\right) \Omega\left(u \times E_{n}\right)\right\} / u^{*} T u
\end{aligned}
$$

for any section vector $u=\sum_{j=1}^{n} b_{j} v_{j}\left(\sum_{j=1}^{n}\left|b_{j}\right|^{2}=1\right)$. (5.9) follows from (5.8) and (5.6).

Remark 5.1. $R_{D}(z ; u) \equiv R_{D}(z ; u, u)=2-\left(\lambda^{(2)}(u)\right)^{2} / \lambda^{(1)} \lambda^{(3)}(u, u)<2$ [2] and $R_{D}(z ; u, v)<2[10]$ are known.

Let $u_{0}$ and $v_{0}$ be any orthogonal vectors such as $u_{0}^{*} T v_{0}=0$; then we have, for $n \geq 2$,

$$
R_{D}\left(z ; u_{0}, v_{0}\right)<1 \text {. }
$$

In a bounded homogeneous domain $D$, the absolute invariant $I_{D}^{(1)}(z, \bar{z})$ under $B H(D)$ (see (4.13)) equals a positive constant in $D$. Therefore, a domain $D$ with $I_{D}^{(1)} \equiv$ constant or a homogeneous domain $D$ satisfies, for any section vector $u$,

$$
C_{D}(z ; u)=-1 \text { and } S_{D}(z)=-n \quad \text { in } D \text {. }
$$

Let $G$ be a bounded domain in $C^{1}$, then we easily have $R_{G}(z ; u, v)=$ $R_{G}(z ; u)=C_{G}(z ; u)=S_{G}(z)$. If $G$ is also homogeneous, we have $R_{G}(z ; u, v)=-1$ in $G$ since $G$ is symmetric by Cartan's theorem and hence is simply connected. 
Theorem 5.2. Let $D$ be a bounded domain in $C^{n}(n \geq 2)$; then we have, for any section vectors $u$ and $v$,

$$
-n+\epsilon+C_{D}(z ; u)<R_{D}(z ; u, v)<1+\epsilon \text { in } D .
$$

In particular, if $D$ is homogeneous, then we have, for any section vectors $u$ and $v$,

$$
\begin{gathered}
-(n+1)+\epsilon<R_{D}(z ; u, v)<1+\epsilon \text { in } D, \\
-n<R_{D}(z ; u)<2 \text { in } D(c f .[10])
\end{gathered}
$$

and there exist some vectors $u^{\prime}$ and $v^{\prime}$ such that

$$
R_{D}\left(z ; u^{\prime}, v^{\prime}\right)<0 \text { in } D .
$$

Proof. Let $A$ be a positive definite Hermitian $n \times n$ matrix and $v^{\prime}=T^{-1 / 2} P$ be a vector with $P^{*} P=1$ i.e., $v$ denotes a vector $\sum_{i=1}^{n} p_{i} v_{i}$, where $P \equiv\left(p_{1}, \ldots, p_{n}\right)^{T}$ and $v_{i}=T^{-1 / 2} M_{i}$ (see (5.4)), then we have $v^{*} A v \leq \mathrm{Sp}_{\mathrm{p}}\left(T^{-1} A\right)$ (inequality for $n \geq 2$ and equality for $n=1$ ). For any vector $v$ with $v^{*} T v=1$, we have (5.12) from (5.7) and (5.8), since we have

$$
(u \times v)^{*} \Omega(u \times v) / u^{*} T u v^{*} T v<\operatorname{Sp}\left\{T^{-1}\left(u^{*} \times E_{n}\right) \Omega\left(u \times E_{n}\right)\right\} / u^{*} T u
$$

from (4.25).

If $D$ is homogeneous, we have (5.13) from (5.12) and (5.11). (5.14) is easily obtained by $\epsilon_{D}(z ; u, u)=1$ in $D$. From (5.5) and (5.11) we have

$$
n\left\{\begin{array}{l}
\inf R_{D}(z ; u, v) \\
u, v
\end{array} \leq-1 \leq n\left\{\sup _{u, v} R_{D}(z ; u, v)\right\}\right.
$$

and hence (5.15).

Theorem 5.3. Let $D$ be a bounded homogeneous domain and $\left(u^{*} \times E_{n}\right) T_{2, D}\left(u \times E_{n}\right)$ be nonnegative definite (resp. positive definite); then we have, for $n \geq 2$,

$$
-1 \leq R_{D}(z ; u, v) \leq 0 \quad\left(\text { resp. }-1<R_{D}(z ; u, v)<0\right) .
$$

Proof. For any section vector $v=T^{-1 / 2} P$ with $P^{*} P=1$, we have $R_{D}(z ; u, v)=-P^{*} Q P / u^{*} T u$, where $Q \equiv T^{-1 / 2}\left(u^{*} \times E_{n}\right) T T_{2, D}\left(u \times E_{n}\right) T^{-1 / 2}$ $=U^{*}\left(\lambda_{1}+\cdots+\lambda_{n}\right) U$ ( $U$ : unitary $n \times n$ matrix and $\left.\lambda_{1} \geq \cdots \geq \lambda_{n} \geq 0\right)$, since $T \equiv T_{D}(z, \bar{z})$ and $\left(u^{*} \times E_{n}\right) T_{2, D}\left(u \times E_{n}\right)$ are positive and nonnegative (resp. positive) definite, respectively. Set $U P=S \equiv\left(s_{1}, \ldots, s_{n}\right)^{T}$, then we have $S^{*} S=1$. Let $D$ be a homogeneous domain with $Q \geq 0$, then it fol- 
lows that $-1 \leq S_{D}(z ; u)=-\mathrm{Sp}(Q) / u^{*} T u=-\Sigma_{i=1}^{n} \lambda_{i} / u^{*} T u$ and thus $\sum_{i=1}^{n} \lambda_{i}$ $=u^{*} T u>0$, i.e., $\lambda_{1}>0$. Hence we get

$$
\begin{aligned}
-1 & \leq-\lambda_{1} / \sum_{i=1}^{n} \lambda_{i} \leq R_{D}(z ; u, v) \\
& =-\sum_{i=1}^{n} \lambda_{i}\left|s_{i}\right|^{2} / \sum_{i=1}^{n} \lambda_{i} \leq-\lambda_{n} / \sum_{i=1}^{n} \lambda_{i} \leq 0 .
\end{aligned}
$$

Example 5.1. Any classical Cartan domain $D$ satisfies that $v^{*}\left(u^{*} \times E_{n}\right) T_{2, D}\left(u \times E_{n}\right) v \geq 0$ for any section vector $v$. Therefore, (5.16) holds in $D$. Let $R(i)(i=\mathrm{I}$, II, III, IV) be the classical Cartan domains (four main types of irreducible bounded symmetric domains). They are homogeneous, and the following hold [1. $]$ :

$$
\begin{aligned}
-2 /(m+n) & \leq R_{R(\mathrm{I})}(z ; u) \leq-2 / m(m+n) \quad(m \geq n \geq 1), \\
-2 /(n+1) & \leq R_{R(\mathrm{II})}(z ; u) \leq-2 / n(n+1), \\
-1 /(n-1) & \leq R_{R(\mathrm{III})}(z ; u) \leq-1 /[n / 2](n-1) \quad(n \geq 2), \\
-2 / n & \leq R_{R(\mathrm{IV})}(z ; u) \leq-1 / n .
\end{aligned}
$$

For the $n$-polydisc $P$ and the unit hypersphere $E$

$$
-1 \leq R_{D}(z ; u) \leq-1 / n \text { and } R_{E}(z ; u)=-2 /(n+1) \text { in } D
$$

hold, but in general $R_{E}(z ; u, v)$ is "not constant" for arbitrary vectors $u$ and $v$.

6. Domains of comparison. The basic tool used here and in the next section is the so-called method of minimum integral [3] or the principle of minimum problems [7].

Principle. Let $\lambda_{A}^{K(m)}(t)$ and $\lambda_{B}^{K(m)}(t)$ be the minimum values defined in $\S 3$ for two domains $A$ and $B$ with $A \subset B$ under the same additional condition $K(m)$ at $t \in A$; then we have

$$
\lambda_{A}^{K(m)}(t) \leq \lambda_{B}^{K(m)}(t) .
$$

Theorem 6.1. Let $A$ and $B$ be domains of comparison of a bounded domain $D(A \subset D \subset B)$ and $\epsilon_{D}(u, v) \equiv \epsilon_{D}(z ; u, v)$; then we have, for $z \in A$, 


$$
\left(1+\epsilon_{B}(u, v)-R_{B}(z ; u, v)\right) / \Lambda_{A B}(u, v) \leq 1+\epsilon_{D}(u, v)-R_{D}(z ; u, v)
$$

$$
\begin{aligned}
& \leq\left(1+\epsilon_{A}(u, v)-R_{A}(z ; u, v)\right) \Lambda_{A B}(u, v), \\
&\left(n+1-C_{B}(z ; u)\right) / \Lambda_{A B}^{1 / 2}(u, u) \leq n+1-C_{D}(z ; u) \\
& \leq\left(n+1-C_{A}(z ; u)\right) \Lambda_{A B}^{1 / 2}(u, u)
\end{aligned}
$$

and

$$
\left(n(n+1)-S_{3}(z)\right) / \Psi_{A B} \leq n(n+1)-S_{D}(z) \leq\left(n(n+1)-S_{A}(z)\right) \Psi_{A B},
$$

where

$$
\begin{aligned}
\Lambda_{A B}(u, v) & \equiv \lambda_{B}^{(2)}(u) \lambda_{B}^{(2)}(v) / \lambda_{A}^{(2)}(u) \lambda_{A}^{(2)}(v) \\
& =k_{A}^{2} u^{*} T_{A} u v^{*} T_{A} v / k_{B}^{2} u^{*} T_{B} u v^{*} T_{B} v
\end{aligned}
$$

and

$$
\Psi_{A B} \equiv \lambda_{B}^{(1)} / \lambda_{A}^{(1)}=k_{A} / k_{B} .
$$

Proof. By Theorem 5.1 and Principle we have

$$
\begin{aligned}
1+\epsilon_{D}(u, v)-R_{D}(z ; u, v) & =\lambda_{D}^{(2)}(u) \lambda_{D}^{(2)}(v) / \lambda_{D}^{(1)} \lambda_{D}^{(3)}(u, v) \\
& \leq\left(1+\epsilon_{A}(u, v)-R_{A}(z ; u, v)\right) \Lambda_{A B}(u, v),
\end{aligned}
$$

etc. Thus we have (6.2), (6.3) and (6.4) by the same procedure.

By Theorem 6.1 and the biholomorphic invariancies of curvatures, we have the following:

Corollary 6.1. (i) If $A$ and $B$ are image domains of the unit hypersphere and $A \subset D \subset B$ holds, then we have, for $z \in A$,

$$
2\left(1-\nu \cdot 1_{A B}(u, u)\right) \leq R_{D}(z ; u) \leq 2\left(1-\nu / \Lambda_{A B}(u, u)\right) .
$$

(ii) If $A$ and $B$ are homogeneous domains of comparison of a bounded domain $D$, then we have, for $z \in A$,

$$
(n+1)\left(1-\nu \Lambda_{A B}^{1 / 2}(u, u)\right) \leq C_{D}(z ; u) \leq(n+1)\left(1-v / \Lambda_{A B}^{1 / 2}(u, u)\right)
$$

and

$$
n(n+1)\left(1-\nu \Psi_{A B}\right) \leq S_{D}(z) \leq n(n+1)\left(1-\nu / \Psi_{A B}\right) .
$$

Here and in the following, $\nu$ denotes $(n+2) /(n+1)$. 
Corollary 6.2. If $A$ and $B$ are hyperspheres of radii $r$ and $R(r<R)$ with the same center at the origin, respectively, and $D(A \subset D \subset B)$ is a homogeneous domain, then we have, for any section vector $u$ and $x \in D$,

$$
2\left(1-\nu(R / r)^{4 n+4}\right) \leq R_{D}(x ; u) \leq 2\left(1-\nu(r / R)^{4 n+4}\right) .
$$

Proof. For such a homogeneous mapping $h(z)$ of $D$ that $h(t)=0$ holds for any fixed point $t \in D$, we have $R_{D}(t ; u)=R_{D}(0 ; v)$, where $v=D_{z} h(t) u$. On the other hand, from (6.5) we have

$$
2\left(1-\nu \Lambda_{A B}(v, v)\right) \leq R_{n}(0 ; v) \leq 2\left(1-\nu / \Lambda_{A B}(v, v)\right) .
$$

The Bergman kernel function $k_{A}(z, \bar{z})$ and the Bergman metric tensor $T_{A}(z, \bar{z})$ of a hypersphere $A \equiv\left\{z|| z \mid<r, z \equiv\left(z_{1}, \ldots, z_{n}\right)^{T}\right\}$ are given by

$$
k_{A}(z, \bar{z})=n ! r^{2} / \pi^{n}\left(r^{2}-z^{*} z\right)^{n+1}
$$

and

$$
T_{A}(z, \bar{z})=(n+1) r^{2}\left(r^{2} \times E_{n}-z z^{*}\right)^{-1} /\left(r^{2}-z^{*} z\right)
$$

as is well known (see [14], [16]). Therefore, we have

$$
\lambda_{A}^{(2)}(0 ; v)=1 / k_{A}(0,0) v^{*} T_{A}(0,0) v=\pi^{n} r^{2 n+2} / n ! v^{*} v
$$

and hence $\Lambda_{A B}(u, u)=\left((R / r)^{2 n+2}\right)^{2}$. Thus we obtain (6.5).

Remark 6.1. The holomorphic sectional curvatures of the classical Cartan domains are always negative as was stated before. All bounded symmetric domains are homogeneous but the converse is not true for $n \geq 4$ (E. Cartan). K. H. Look gave an example of a homogeneous but nonsymmetric domain $D$ having a section $u$ such that $R_{D}(z ; u)$ has a positive value, which is the negative solution on the Hua's conjecture. For any homogeneous domain $D$, which satisfies $A \subset D \subset B$ and $(R / r)^{4 n+4} \leq \nu$ in Corollary 6.2, we have $R_{D}(z ; u) \leq 0$ for any $u$ in $D$.

7. Asymptotic boundary behaviors of curvatures. Now, we shall study the behaviors of curvatures about a boundary point of a bounded domain $D$ with a sort of convexity in $C^{n}$ using the domains of comparison of $D$.

Definition 7.1. Let $D$ be a domain in $C^{n}$. Suppose that there exists an analytic change of coordinates, one-to-one in a neighborhood $\Gamma(\Gamma \supset D)$ of a boundary point $P \in \partial D$, so that, with respect to this change of coordinates, $D \rightarrow \Delta, P \rightarrow Q=\{0\}(Q \in \partial \Delta)$ and 


$$
\Delta=\left\{z \mid z_{1}+\bar{z}_{1}>z^{*} z+o\left(z^{*} z\right)\right\}
$$

in the neighborhood of $Q=\{0\}$. Then $\Delta$ and also the original domain $D$ are said to be strictly pseudoconvex globally representable (simply SPCGR) at $Q$ and also at $P$, respectively. We call the new coordinates "normal" coordinates and the analytic hypersurface $z_{1}=0$ (with respect to the normal coordinates) is called the normal analytic hypersurface (simply NAH) [4], [9].

If $D \equiv\left\{z \mid \phi(z, \bar{z})<0, \phi \in C^{2}\right.$-class in a neighborhood of $\bar{D}, \operatorname{grad}(\phi)$ $\neq 0$ on $\partial D\}$ in $C^{n}$ is a strictly pseudoconvex domain in the sense of Levi at a point $Q=\{0\} \in \partial D$, i.e., $\phi$ satisfies $L(\phi(Q)) \equiv z^{*}\left(\partial^{2} \phi(Q) / \partial z^{*} \partial z\right) z>0$ when $(\partial \phi(Q) / \partial z) z=0$ and $z \neq 0$, then by the Taylor's expansion of $\phi$ at $Q=\{0\}$ and by suitable changes of coordinates (properly affine in $C^{n}$ and biholomorphic in a neighborhood of $\bar{D}$ ), we have the image domain of the type of (7.1) (see [9, Theorem 3.5.1 and its proof]). Therefore, any strictly pseudoconvex domain (in the sense of Levi) with one-to-one "normal" analytic change of coordinates is a SPCGR domain. If $D$ is a SPCGR domain, for the sake of estimates on curvatures, we can use $\Delta$ in (7.1) instead of $D$ from the beginning, since curvatures are biholomorphically invariant.

The hypersphere

(7.2) $R_{\delta} \equiv\left\{\zeta \mid \zeta_{1}+\bar{\zeta}_{1}>\zeta^{*} \zeta+\delta \zeta^{*} \zeta, \delta(-1<\delta<1)\right.$ : real constant number $\}$

is biholomorphically equivalent to the unit hypersphere $E \equiv\{z|| z \mid<1\}$ under the transformation

$$
T_{\delta}: z=(1+\delta) \zeta-(1,0, \ldots, 0)^{T}
$$

B. L. Chalmers [4] has given the domains of comparison $R_{-\epsilon}^{\alpha \beta}$ and $R_{\epsilon}^{a^{\prime} \beta^{\prime}}(\epsilon>0)$ for a strictly $(p, q)$ pseudoconvex globally representable domain $D$ with the normal analytic hypersurface $h \equiv\left\{\zeta \mid \zeta_{1}=0\right\}$ lying entirely outside $D$. In the following, we shall treat a strictly $(1, n)$ pseudoconvex (usual pseudoconvex) globally representable domain (7.1) with the normal analytic hypersurface $b$ lying entirely outside itself, which is called a SPCGR-NAH domain at $Q$.

$R_{-\epsilon}^{\alpha \beta}$ and $R_{\epsilon}^{a^{\prime} \beta^{\prime}}$ are equivalent to the hypersphere $R_{-\epsilon}$ and $R_{\epsilon}$ (see (7.2)) under biholomorphic mappings

$$
W: z_{1}=\zeta_{1} /\left(1-\alpha \zeta_{1}\right), z_{k}=\zeta_{k}\left\{1+(\beta-\alpha) \zeta_{1}\right\} /\left(1-\alpha \zeta_{1}\right),
$$

$$
k=2, \ldots, n,
$$


and

$$
\begin{array}{r}
W^{\prime}: z_{1}=\zeta_{1} /\left(1+a^{\prime} \zeta_{1}\right), z_{k}=\zeta_{k}\left(1+\alpha^{\prime} \zeta_{1}\right) /\left\{1+\left(a^{\prime}+\beta^{\prime}\right) \zeta_{1}\right\}, \\
k=2, \ldots, n,
\end{array}
$$

respectively. In particular, for sufficiently large numbers $\alpha, \beta, \alpha^{\prime}$ and $\beta^{\prime}$, we have

$$
R_{\epsilon}^{a^{\prime} \beta^{\prime}} \subset \Delta \subset R_{-\epsilon}^{\alpha \beta}
$$

where $\Delta$ denotes a SPCGR-NAH domain at $Q=\{0\}$ [4].

Definition 7.2. We shall write $\lim _{\zeta \rightarrow 0}^{A}$, or sometimes simply $\lim ^{A}$, to indicate a limit is being taken as $\zeta \rightarrow 0$ in the set $0<a<\operatorname{Re}\left(\zeta_{1}\right) /|\zeta|$ (a: positive constant number) and say $\zeta \rightarrow 0$ via an $A$-approach after Chalmers [4].

Lemma 7.1. For a hypersphere $R_{\delta}(0<\delta<1)$ we have

$$
\lim _{\zeta \rightarrow 0}^{A}\left(\zeta_{1}+\bar{\zeta}_{1}\right)^{n+1} k_{R \delta}(\zeta, \bar{\zeta})=n !(1+\delta)^{n-1} / \pi^{n}
$$

and for any constant nonzero vector $u \equiv\left(u_{1}, \ldots, u_{n}\right)^{T}$

$$
\lim _{\zeta \rightarrow 0}^{A}\left(\zeta_{1}+\bar{\zeta}_{1}\right)^{2} u^{*} T_{R_{\delta}}(\zeta, \bar{\zeta}) u= \begin{cases}(n+1)\left|u_{1}\right|^{2} & \text { for } u_{1} \neq 0 \\ (n+1)(1+\delta)|u|^{2} & \text { for } u_{1}=0\end{cases}
$$

Proof. Let $E$ be a unit disc in $C^{n}$. Since $k_{E}(z, \bar{z})=n ! / \pi^{n}\left(1-z^{*} z\right)^{n+1}$ (6.9) and $k_{R_{\delta}}(\zeta, \bar{\zeta})=k_{E}(z, \bar{z})\left|J_{z}(\zeta)\right|^{2}=k_{E}(z, \bar{z})(1+\delta)^{2 n}$ for (7.3); then we have

$$
k_{R \delta}(\zeta, \bar{\zeta})=n !(1+\delta)^{n-1} / \pi^{n} \Lambda_{\delta}^{n+1}
$$

where $1-z^{*} z=(1+\delta) \Delta_{\delta}$ and $\Delta_{\delta}=\zeta_{1}+\bar{\zeta}_{1}-(1+\delta)|\zeta|^{2}$. Noting that $\lim _{\zeta \rightarrow 0^{\Delta}}^{A} \Delta_{-\epsilon} / \Delta_{\epsilon}=1$, we obtain (7.7).

Let us set $z \equiv U(z)(\rho, 0, \ldots, 0)^{T}$, where $U(z)=U(z(\zeta))$ denotes a unitary matrix and $\rho(\rho>0) \rightarrow 1$ (for $z \rightarrow(-1,0, \ldots, 0)^{T}$ ) is equivalent to $\zeta \rightarrow 0$ under (7.3). If we set $U^{*}(z) u=U^{*}(z(\zeta)) u \equiv v \equiv\left(v_{1}, \ldots, v_{n}\right)^{T}$ and $\lim ^{A} v=v_{0} \equiv\left(v_{1}^{0}, \ldots, v_{n}^{0}\right)^{T}$, then we have $|v|=\left|v_{0}\right|=|u|$ and $v_{1}^{0}=-u_{1}$, because $z^{*} u=(\rho, 0, \ldots, 0) U^{*}(z) u=\rho v_{1} \rightarrow v_{1}^{0}$ and

$$
z^{*} u=\left\{(1+\delta) \zeta^{*}-(1,0, \ldots .0)\right\} u=(1+\delta) \zeta^{*} u-u_{1} \rightarrow-u_{1}
$$


for an $A$-approach. Further, we have, from (6.10) and $T_{R_{\delta}}(\zeta, \bar{\zeta})=$ $\left(D_{\zeta} z\right) * T_{E}(z, \bar{z}) D_{\zeta} z$

$$
T_{R_{\delta}}(\zeta, \bar{\zeta})=(n+1) U(z)\left\{1+(1+\delta) \Delta_{\delta} \dot{+} \cdot \dot{+}(1+\delta) \Delta_{\delta}\right\} U^{*}(z) / \Delta_{\delta}^{2}
$$

and thus

$$
u^{*} T_{R_{\delta}}(\zeta, \bar{\zeta}) u=(n+1) P_{\delta} / \Delta_{\delta}^{2}, \quad P_{\delta}=\left|v_{1}\right|^{2}+(1+\delta) \Delta_{\delta} \sum_{i=2}^{n}\left|v_{i}\right|^{2} .
$$

Since we easily have $\lim _{\zeta \rightarrow 0}^{A} P_{\delta}=\left|u_{1}\right|^{2}$ and thus (7.8) for $u_{1} \neq 0$.

If $u_{1}=0$, we have

$$
\lim ^{A} P_{\delta} /\left(\zeta_{1}+\bar{\zeta}_{1}\right)=(1+\delta) \sum_{i=2}^{n}\left|v_{i}^{0}\right|^{2}=(1+\delta)|u|^{2},
$$

because we have $z^{*} u=\rho v_{1}=(1+\delta) \zeta^{*} u-u_{1}=(1+\delta) \Sigma_{i=2}^{n} \bar{\zeta}_{i} u_{i}$ for $u_{1}=0$, and hence

$$
\begin{array}{r}
P_{\delta}=\left|(1+\delta) \sum_{i=2}^{n} \bar{\zeta}_{i} u_{i} / \rho\right|^{2}+(1+\delta)\left(\zeta_{1}+\bar{\zeta}_{1}-(1+\delta)|\zeta|^{2}\right) \sum_{i=2}^{n}\left|v_{i}\right|^{2} \\
=(1+\delta)\left(\zeta_{1}+\bar{\zeta}_{1}\right) \sum_{i=2}^{n}\left|v_{i}\right|^{2}+o\left(\zeta_{1}+\bar{\zeta}_{1}\right)
\end{array}
$$

follows from

$$
\left|(1+\delta) \sum_{i=2}^{n} \bar{\zeta}_{i} u_{i} / \rho\right|^{2} /\left(\zeta_{1}+\bar{\zeta}_{1}\right) \leq(1+\delta)^{2}|u|^{2}|\zeta|^{2} / \rho^{2}\left(\zeta_{1}+\bar{\zeta}_{1}\right) \rightarrow 0
$$

and $(1+\delta)^{2}|\zeta|^{2} \Sigma_{i=2}^{n}\left|\nu_{i}\right|^{2} /\left(\zeta_{1}+\bar{\zeta}_{1}\right) \rightarrow 0$ for an $A$-approach. Now, noting (7.7), $\lim ^{A} \Delta_{-\epsilon} / \Delta_{\epsilon}=1$ and $\left.\lim ^{A}\right\lrcorner_{\delta} /\left(\zeta_{1}+\bar{\zeta}_{1}\right)=1$, we obtain (7.8) for $u_{1}=0$.

Lemma 7.2. Setting $R_{\epsilon}^{a^{\prime} \beta^{\prime}}=A$ and $R_{-\epsilon}^{\alpha \beta}=B$, we have

$$
\text { (7.9) } \lim _{\zeta \rightarrow 0}^{A} \Psi_{A B}(\zeta, \bar{\zeta})=\lim _{\zeta \rightarrow 0}^{A} k_{A}(\zeta, \bar{\zeta}) / k_{B}(\zeta, \bar{\zeta})=\{(1+\epsilon) /(1-\epsilon)\}^{n-1}
$$

and for any constant nonzero vector $u \equiv\left(u_{1}, \ldots, u_{n}\right)^{T}$ 


$$
\lim _{\zeta \rightarrow 0}^{A} \Lambda_{A B}^{1 / 2}(\zeta, \bar{\zeta})=\lim _{\zeta \rightarrow 0}^{A} k_{A}(\zeta, \bar{\zeta}) u^{*} T_{A}(\zeta, \bar{\zeta}) u / k_{B}(\zeta, \bar{\zeta}) u^{*} T_{B}(\zeta, \bar{\zeta}) u
$$

$$
= \begin{cases}\{(1+\epsilon) /(1-\epsilon)\}^{n-1} & \text { for } u_{1} \neq 0, \\ \{(1+\epsilon) /(1-\epsilon)\}^{n} & \text { for } u_{1}=0,\end{cases}
$$

where $\epsilon$ denotes an arbitrary constant number in the interval $(0,1)$.

Proof. By the relative invariancies of $k_{D}$ and $T_{D}$ under $B H(D)$, it suffices to prove that (7.9) and (7.10) for $R_{\epsilon}$ and $R_{-\epsilon}$ in place of $A$ and $B$ are shown, respectively, since we have $d \zeta / d z \rightarrow E_{n}$ and $\left|J_{\zeta}(z)\right| \rightarrow 1$ for each mapping (7.4) or (7.5) via an $A$-approach. Therefore, (7.9) and (7.10) are obtained by Lemma 7.1.

Theorem 7.1. Let $D$ be a bounded SPCGR-NAH domain at $Q$; then we have, for any constant nonzero vector $u \equiv\left(u_{1}, \ldots, u_{n}\right)^{T}$,

$$
\lim _{z \rightarrow Q}{ }^{A} R_{D}(z ; u)=-2 /(n+1)
$$

(cf. Bergman [3] for $n=1$, Fuks [7] for $n=2$ ),

$$
\lim _{z \rightarrow Q}^{A} C_{D}(z ; u)=-1
$$

(cf. Fuks [8] for $n=2$ ) and

$$
\lim _{z \rightarrow Q}^{A} S_{D}(z)=-n
$$

Proof. Using Corollary 6.1, Lemma 7.2, (5.11) and (5.17), we conclude (7.11), (7.12) and (7.13), since $R_{-\epsilon}^{a \beta}, R_{\epsilon}^{a^{\prime} \beta^{\prime}}, R_{-\epsilon}$ and $R_{\epsilon}$ are biholomorphically equivalent to the unit hypersphere and $\epsilon$ can be taken as sinall as we need by taking sufficiently large numbers $\alpha, \beta, \alpha^{\prime}$ and $\beta^{\prime}$.

Now, we turn to compose another sort of domains of comparison, which is an inmediate extension of domains of comparison due to Bergman for $n=1[3, \mathrm{p} .38]$.

The set $U(r) \equiv\left\{z|| z_{1}-\left.r\right|^{2}+\sum_{i=2}^{n}\left|z_{i}\right|^{2}<r^{2}, r\right.$ : positive constant $\}$ and $B(r) \equiv\left\{z|| z_{1}+\left.r\right|^{2}>r^{2}+\sum_{i=2}^{n}\left|z_{i}\right|^{2}, r\right.$ : positive constant $\}$ are biholomorphically equivalent to the unit hypersphere $E$ under the mappings

$$
z=\zeta / r-(1,0, \ldots, 0)^{T}
$$

and

$$
z=\zeta /\left(\zeta_{1}+r\right)-(1,0, \ldots, 0)^{T}
$$


whose Jacobian determinants tend to $r^{-n}$ and $-r^{-n}$ for $\zeta \rightarrow 0$, respectively. $B(r)$ is similar to a Siegel domain of the second kind. If we consider the sections $U(r ; t)$ and $B(R ; t)$ restricted by the counter surface $\sum_{i=2}^{n}\left|\zeta_{i}\right|^{2}=r^{2} t(0 \leq t<1)$, we have

$$
U(r ; t)=\left\{\zeta_{1} \| \zeta_{1}-r \mid<\sqrt{1-t}\right\} \subset 3(R ; t)=\left\{\zeta_{1} \| \zeta_{1}+R \mid>\sqrt{R^{2}+r^{2} t}\right\}
$$

and thus $U(r) \subset B(R)$ and $\partial U(r) \cap \partial B(R)=\{0\}$ for $R \geq r$.

By the same procedure in the proof of Lemmas 7.1 and 7.2, we have the following Lemma 7.3 and Theorem 7.2.

Lemma 7.3. If $R \geq r$, we have, for $n \geq 1$,

$$
\lim _{\zeta \rightarrow 0}^{A} \lambda_{B(R)}^{(1)} / \lambda_{U(r)}^{(1)}=\lim _{\zeta \rightarrow 0}^{A} \lambda_{U(R)}^{(1)} / \lambda_{U(r)}^{(1)}=(R / r)^{n-1}
$$

and for any constant nonzero vector $u \equiv\left(u_{1}, \ldots, u_{n}\right)^{T}$

$$
\begin{aligned}
\lim _{\zeta \rightarrow 0}^{A} \lambda_{B(R)}^{(2)}(u) / \lambda_{U(r)}^{(2)} & =\lim _{\zeta \rightarrow 0}^{A} \lambda_{U(R)}^{(2)}(u) / \lambda_{U(r)}^{(2)}(u) \\
& = \begin{cases}(R / r)^{n-1} & \text { for } u_{1} \neq 0, \\
(R / r)^{n} & \text { for } u_{1}=0 .\end{cases}
\end{aligned}
$$

Theorem 7.2. Let $D$ be a bounded domain which has domains $U(r)$ and $B(r)(U(r) \subset D \subset B(r))$ of comparison, then we have the same results as in Theorem 7.1.

Example 7.1. (i) Let $H$ be a Hartogs domain (complete multicircular domain with center at $\left.(\psi(0), 0)^{T}\right)\left\{z|| z_{1}-\psi(0)\left|<\psi\left(\left|z_{2}\right|\right),\right| z_{2} \mid<r, r>0\right.$, $\psi(\rho) \in C^{2}$-class and $\left.\psi(0)>0, \psi^{\prime}(0)=0, \psi^{\prime \prime}(0)<0\right\}$. Set $\Psi(z, \bar{z}) \equiv$ $\left|z_{1}-\psi(0)\right|^{2}-\psi^{2}\left(\left|z_{2}\right|\right)(H \equiv\{z \mid \Psi<0\})$. Then we have the Levi determinant $L(\Psi)=-\psi^{2}(0) \lambda^{\prime}(0)$, where $\lambda\left(\rho^{2}\right)=\psi^{2}(\rho)$ and $\lambda^{\prime}(0)$ denotes $d \lambda(x) /\left.d x\right|_{x=0^{\circ}}$ Since $\lambda^{\prime}(0)=\psi(0) \psi^{\prime \prime}(0)<0$, then $H$ is strictly pseudoconvex at 0 . As $H$ is expressed as

$$
\left\{\left.z\left|z_{1}+\bar{z}_{1}>\left(\left|z_{1}\right|^{2}-\lambda^{\prime}(0)\left|z_{2}\right|^{2}\right) / \psi(0)+o\right| z\right|^{2}\right\}
$$

(about the origin), $H$ is a SPCGR-NAH domain at 0 . Therefore, Theorem 7.1 holds in this case, i.e., $\lim ^{A} R_{H}(z ; u)=-2 / 3, \lim ^{A} C_{H}(z ; u)=-1$ and $\lim ^{A} S_{H}(z)=-2$. 
(ii) Let us set $H^{\prime} \equiv\left\{z|| z_{1}-\psi(0)\left|<\psi\left(\left|z_{2}\right|\right),\right| z_{2} \mid<r, r>0, \psi(\rho)\right.$ $(0 \leq \rho \leq r)$ is a decreasing real valued continuous function which satisfies $\psi(0)-a+\sqrt{a^{2}-\rho^{2}} \leq \psi(\rho) \leq \psi(0)+a-\sqrt{a^{2}+\rho^{2}}$ and $\left.\psi(0)>a>r\right\}$. Then $H^{\prime}$ has the domains of comparison: $U(a)=\left\{z|| z_{1}-\left.a\right|^{2}+\left|z_{2}\right|^{2}<a^{2}\right\}$ and $B(a)=\left\{z|| z_{1}+\left.a\right|^{2}>a^{2}+\left|z_{2}\right|^{2}\right\}$, since $\psi(0)-a+\sqrt{a^{2}-\rho^{2}} \leq \psi(\rho)$ and $\sqrt{a^{2}+\rho^{2}}+\psi(\rho) \leq \psi(0)+a$ imply $U(a) \subset H^{\prime}$ and $H^{\prime} \subset B(a)$, respectively, and $\partial U(a) \cap \partial B(a) \cap \partial H^{\prime}=\{0\}$ is evident. Hence from Theorem 7.2 we have the same results as in (i).

Theorem 7.3. If $A=U(r)$ and $B=U(R)$ (or $B=B(R)$ ) are domains of comparison such that $A \subset D \subset B$ and $\partial A \cap \partial B \cap \partial D=\{0\}$ for $r \leq R$, then we have, for any nonzero vector $u \equiv\left(u_{1}, \ldots, u_{n}\right)^{T}$,

$$
2\left\{1-\nu(R / r)^{2(n-1)}\right\} \leq \lim _{\zeta \rightarrow 0}^{A} R_{D}(\zeta ; u) \leq 2\left\{1-\nu(r / R)^{2 n}\right\},
$$

$$
(n+1)\left\{1-\nu(R / r)^{n-1}\right\} \leq \lim _{\zeta \rightarrow 0}^{A} C_{D}(\zeta ; u) \leq(n+1)\left\{1-\nu(r / R)^{n}\right\}
$$

and

(7.20) $n(n+1)\left\{1-\nu(R / r)^{n-1}\right\} \leq \lim _{\zeta \rightarrow 0}^{A} S_{D}(z) \leq n(n+1)\left\{1-\nu(r / R)^{n-1}\right\}$.

Proof. From Lemma 7.3 and Corollary 6.1, we have the results.

8. On the Ricci scalar curvature.

Theorem 8.1. In a bounded domain $D$ we consider the quantity

$J_{D, p}(z, \bar{z}) \equiv J_{D,(p, 1)}(z, \bar{z})=\operatorname{det}\left(k_{D}^{p}(z, \bar{z}) \times T_{D}(z, \bar{z})\right) \quad($ see $(4.22))$.

(i) For $p \geq(n+1) / n$, which is the case that the metric $d s_{D, p}^{2} \equiv d s_{D,(p, 1)}^{2}$ can be defined (see Corollary 4.2), it holds that $\Delta \log J_{D, p}(z, \bar{z})>0$ for $z \in D$ and there is no fixed point $z^{0} \in D$ such that $J_{D, p}(z, \bar{z}) \leq$ $J_{D, p}\left(z^{0}, \bar{z}^{0}\right)$ for $z \in D$, where $\Delta$ denotes the Laplace-Beltrami operator: Sp $T_{D}^{-1} D_{z}^{*} D_{z}$

(ii) If there exists a maximal point $z^{0} \in D$ such that $J_{D, p}(z, \bar{z}) \leq$ $J_{D, p}\left(z^{0}, \bar{z}^{0}\right)$ for $z \in D$, then $p$ must be smaller than $(n+1) / n$.

Proof. Since $S_{D}(z)<n(n+1)$ holds for a bounded domain $D$, $\Delta \log J_{D, p}=\operatorname{Sp}_{\mathrm{P}}\left\{T_{D}^{-1}\left(p n T_{D}-\left(R_{\bar{\alpha} \beta}\right)\right\}=p n^{2}-S_{D}(z)>p n^{2}-n(n+1) \geq 0\right.$ 
for $p \geq(n+1) / n$. If there exists a point $z^{0} \in D$ such that $J_{D, p}(z, \bar{z}) \leq$ $J_{D, p}\left(z^{0}, \bar{z}^{0}\right)$ for $z \in D$, then by the theorem of E. Hopf (see [22]) we obtain $J_{D, p} \equiv$ constant. Hence $p n T_{D}-\left(R_{\bar{\alpha} \beta}\right)=0$ for $z \in D$ follows and thus $S_{D}(z)=p n^{2} \geq n(n+1)$ for $p \geq(n+1) / n$, which is contradictory to (5.9). The proof of (ii) is clear.

Remark 8.1. [12, Theorem 3.10] says that in a bounded domain $D$, if there exists $z^{0} \in D$ such that $J_{D}(z, \bar{z}) \leq J_{D}\left(z^{0}, \bar{z}^{0}\right)$ for $z \in D$, where $J_{D} \equiv$ $k_{D}^{n+1} \operatorname{det} T_{D}\left(=J_{D,(n+1) / n}\right)$, then we have $J_{D}(z, \bar{z})=$ constant and $S_{D}(z)=$ $n(n+1)$. But this conclusion contradicts (5.9). Therefore, it seems to be faulty. This is also an impossible case of Theorem 8.1 (i) for $p=(n+1) / n$.

For $p=-1 / n$, we have $J_{D,-1 / n}=\operatorname{det} T_{D} / k_{D} \equiv I_{D}^{(1)}(z, \bar{z})$ which is a biholomorphically absolute invariant (see (4.13)). Thus the following theorem is an extension of [12, Theorem 3.9], which is obtained immediately by setting $p=-1 / n$ in Theorem 8.1.

Theorem 8.2. In a bounded domain $D$, let $S_{D}(z) \geq s_{0}$ (resp. $\left.S_{D}(z) \leq s_{0}\right)$ for $z \in D$, where $s_{0}$ is such a constant number that $s_{0}<n(n+1)$. If for a real number $p \leq s_{0} / n^{2}$ (resp. $\left.p \geq s_{0} / n^{2}\right) J_{D, p}(z, \bar{z}) \geq J_{D, p}\left(z^{0}, \bar{z}^{0}\right)$ (resp. $\left.J_{D, p}(z, \bar{z}) \leq J_{D, p}\left(z^{0}, \bar{z}^{0}\right)\right)$ in $D$ holds for a fixed point $z^{0} \in D$, then we have $S_{D}(z)=s_{0}$ in $D$.

Proof. If $S_{D}(z) \geq s_{0}$ for $z \in D$ and $p \leq s_{0} / n^{2}$, we have $\Delta \log J_{D, p}(z, \bar{z})$ $=p n^{2}-S_{D}(z) \leq p n^{2}-s_{0} \leq 0$ for $z \in D$. Therefore, if $J_{D, p}(z, \bar{z}) \geq$ $J_{D, p}\left(z^{0}, \bar{z}^{0}\right)$ holds for $z \in D$, then from the theorem of E. Hopf we obtain $J_{D, p}(z, \bar{z})=$ constant in $D$ and thus $S_{D}(z)=p n^{2} \leq s_{0}$. On the other hand, $S_{D}(z) \geq s_{0}$ holds from the hypothesis. Then we have $S_{D}(z)=s_{0}$ in $D$.

Theorem 8.3. In a bounded homogeneous domain $D$, if $J_{D, p}(z, \bar{z}) \geq$ $J_{D, p}\left(z^{0}, \bar{z}^{0}\right)$ (resp. $\left.J_{D, p}(z, \bar{z}) \leq J_{D, p}\left(z^{0}, \bar{z}^{0}\right)\right)$ holds in $D$, then we have $J_{D, p}(z, \bar{z})=$ constant in $D$ when and only when $p=-1 / n$, i.e., $J_{D, p}(z, \bar{z}) \equiv$ $I_{D}^{(1)}(z, \bar{z})$ (see (4.13)).

Proof. From (5.11) we have $S_{D}(z)=-n$. Therefore, if $J_{D, p}(z, \bar{z})=$ constant, we have $\Delta \log J_{D, p}(z, \bar{z})=p n^{2}+n=0$ and thus $p=-1 / n$. On the other hand, if $p=-1 / n$, we have $\Delta \log J_{D, p}(z, \bar{z})=0$ in $D$. Using the hypothesis and the theorem of Hopf, we obtain $J_{D, p}(z, \bar{z})=$ constant.

Example 8.1. In the case of the first type $R(\mathrm{I})$ of the classical Cartan domains, which are homogeneous domains, we hạve

$$
J_{R(\mathrm{I}), p}(z, \bar{z})=k_{R(\mathrm{I})}^{p m n} \operatorname{det} T_{R(\mathrm{I})}=(m+n)^{m n} / V \operatorname{det}\left(E_{m}-z^{*} z\right)^{(m+n)(p m n+1)}
$$


$(\operatorname{dim} R(\mathrm{I})=m n)$, where $V$ denotes the Euclidean volume of $R(\mathrm{I})$. Therefore, $J_{R(\mathrm{I}), p}(z, \bar{z})=$ constant $=(m+n)^{m n} / V$ holds when and only when $p=-1 / m n$ (see [16]).

Theorem 8.4. Let $D$ be a bounded domain in $C^{2}$, whose Levi-expression $L(\phi)\left(\phi \in C^{2}\right.$-class) is positive at every point on $D$ and let $I_{D}^{(1)}(z, \bar{z}) \equiv \operatorname{det} T_{D}(z, \bar{z}) / k_{D}(z, \bar{z})$ be nonconstant. If there exists a point $z^{0} \in D$ such that $I_{D}^{(1)}\left(z^{0}, \bar{z}^{0}\right)>9 \pi^{2} / 2$ (resp. $\left.I_{D}^{(1)}\left(z^{0}, \bar{z}^{0}\right)<9 \pi^{2} / 2\right)$, then $S_{D}(z)$ cannot be bounded by -2 from above (resp. below).

Proof. In a bounded homogeneous domain $G, I_{G}^{(1)}(z, \bar{z})=$ constant in G. Therefore, the domain $D$ mentioned here is a nonhomogeneous domain. By the result of Bergman [3], $I_{D}^{(1)}(z, \bar{z})$ must assume its maximum (or minimum) in $D$ with $L(\phi)>0$. If there exists a point $z^{0} \in D$ such that $I_{D}^{(1)}\left(z^{0}, \bar{z}^{0}\right)>9 \pi^{2} / 2, I_{D}(z, \bar{z})$ must have its maximum in $D$. In this case, if $\Delta \log I_{D}^{(1)}(z, \bar{z})=-2-S_{D}(z) \geq 0$ in $D$, we have, by the theorem of Hopf, $I_{D}^{(1)}(z, \bar{z})=$ constant in $D$. This is a contradiction. Therefore, $S_{D}(z)$ cannot be bounded by -2 from above.

9. Reproducing kernel functions of subspaces. Recently, B. L. Chalmers [5] has shown that the Riesz representation of any bounded linear functional in a Hilbert space with kernel function is obtained by operating with the linear functional on the kernel function itself and that, using this representation, one can display, in terms of the kernel function of the original space, the kernel function of any closed subspace defined as the intersection of the null spaces of at most countably many bounded linear functionals. In [5] he gives the following

Proposition 9.1. Let $k_{D}(z, \bar{w})$ be the reproducing kernel function of a bounded domain $D$ and $\mathcal{L}_{(m)} \equiv\left(\mathscr{L}_{1}, \ldots, \mathcal{L}_{m}\right)$ be any bounded linear functionals with respect to $z$ in $D$ which are linearly independent. Then the kernel function of a subspace $\mathcal{\perp}_{(m)}^{2}(D)=\left\{f \in \mathcal{L}^{2}(D) \mid \mathcal{L}_{(m)} f=K(m) \equiv(0, \ldots, 0)\right\}$ is given by

$$
\begin{aligned}
& k_{D, m}(z, \bar{w})=\operatorname{det}\left(\begin{array}{ll}
k_{D}(z, \bar{w}), & \mathscr{L}_{(m)} k_{D}(z, \bar{w}) \\
\mathscr{L}_{(m)}^{*} k_{D}(z, \bar{w}), & \mathfrak{L}_{(m)}^{*} \mathcal{L}_{(m)} k_{D}(z, \bar{w})
\end{array}\right) \\
& \text { - }\left(\operatorname{det} \mathscr{L}_{(m)}^{*} \mathscr{L}_{(m)}{ }^{k}{ }_{D}(z, \bar{w})\right)^{-1} \text {, }
\end{aligned}
$$

where $\mathcal{\complement}_{(m)}^{*} \varrho_{(m)}{ }^{k}{ }_{D}(z, \bar{w})=\left(\bigodot_{(m)}\left(\complement_{(m)}{ }^{k}{ }_{D}(z, \bar{w})\right)^{*}\right)^{*}[5],[18]$. 
The kernel function $k_{D}(z, \bar{w})$ has interesting minimalities as is well known (see (3.12)). We shall give another expression of $k_{D, m}(z, \bar{w}) \equiv$ $k_{m}(z, \bar{w})$ as a minimizing function and show a sort of minimality of it by making use of the general minimum problem for $Q(z, \bar{z}) \equiv Q(z)=k_{D}(z, \bar{w})$.

Theorem 9.1. For any fixed point $w \in D$, under the additional condition $Q(z, \bar{z}) \equiv Q(z)=k_{D}(z, \bar{w})$ and $\mathscr{L}_{(m)} f=K(m) \equiv(0, \ldots, 0)$, we have the minimizing function

$$
M_{D, k}^{(m)}(z, w)=k_{D}(z, \bar{w})-\phi_{D}^{*}(w) \Phi_{m}\left(\Phi_{m}^{*} \Phi_{m}\right)^{-1} \Phi_{m}^{*} \phi_{D}(z) \in \mathscr{L}_{(m)}^{2}(D),
$$

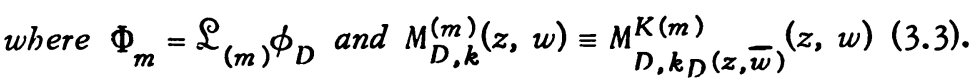

The function $M_{D, k}^{(m)}(z, w)$ coincides with the reproducing kernel function $k_{m}(z, \bar{w}) \in \mathcal{L}_{(m)}^{2}(D)$ and equals the minimum value $\lambda_{D, k_{D}(z, \bar{w})}^{K(w)}$ at (3.4) with $K(m) \equiv(0, \ldots, 0)$. Further $M_{D, k}^{(m)}(w, w)=k_{m}(w, \bar{w}) \leq k_{D}(w, \bar{w})$ holds.

Proof. In Theorem 3.1 if we set $Q(z, \bar{z}) \equiv k_{D}(z, \bar{w}) \in \mathscr{L}^{2}(D)$ (w: fixed) and $\mathscr{L}_{(m)} f=K(m)=(0)$, we have, from (3.3),

$$
M_{D, k}^{(m)}(z, w)=\left\{B-B \Phi_{m}\left(\Phi_{m}^{*} \Phi_{m}\right)^{-1} \Phi_{m}^{*}\right\} \phi_{D}(z) \in \mathscr{L}_{(m)}^{2}(D),
$$

where $B=\int_{D} k_{D}(\zeta, \bar{w}) \phi_{D}^{*}(\zeta) \omega_{\zeta}=\int_{D} \phi_{D}^{*}(w) \phi_{D}(\zeta) \phi_{D}^{*}(\zeta) \omega_{\zeta}=\phi_{D}^{*}(w)$. Noting $\phi_{D}^{*}(w) \phi_{D}(z) \equiv k_{D}(z, \bar{w})$, we have (9.2).

Since, for any $f(z) \in \mathcal{Q}_{(m)}^{2}(D)$,

$$
\int_{D} f(\zeta)\left(\mathfrak{\complement}_{(m)}^{*} k_{D}(\zeta, \bar{w})\right)^{*} \omega_{\zeta}=\int_{D} f(\zeta)\left(\Phi_{m}^{*} \phi_{D}(\zeta)\right)^{*} \omega_{\zeta}=\mathfrak{L}_{(m)} f=0
$$

follows from the Riesz's theorem, then we have

$$
\int_{D} f(z) M_{D, k}^{(m)^{*}}(z, w) \omega_{z}=\int_{D} f(z) k_{D}(w, \bar{z}) \omega_{z}+0=f(w),
$$

which shows that $M_{D, k}^{(m)}(z, w)$ has the reproducing property in $\mathcal{L}_{(m)}^{2}(D)$. And further, $M_{D, k}^{(m)}(z, w)$ coincides with $k_{m}(z, \bar{w})$ by means of (9.1), since, in general, $\operatorname{det}\left(\begin{array}{ll}a & B \\ C & D\end{array}\right)(\operatorname{det} D)^{-1}=a-B D^{-1} C$ holds for a scalar $a$ and a nonsingular matrix $D$. Last parts of the theorem are easily obtained by (3.3) and (3.4).

Remark 9.1. If we set

$$
\mathfrak{L}_{(m)} \equiv\left(\mathfrak{L}_{r(1), t_{1}}, \ldots, \mathfrak{L}_{r(m), t_{m}}\right),
$$


where

$$
\mathfrak{L}_{r(k), t_{k}} f \equiv d^{r(k)} f(z) /\left.d z^{r(k)}\right|_{z=t_{k}} \quad(k=1, \ldots, m)
$$

and

$$
d^{r(k)} / d z^{r(k)} \equiv \partial^{r(k)} / \partial z_{1}^{r(k, 1)} \cdots \partial z_{n}^{r(k, n)}
$$

with $\sum_{i=1}^{n} r(k, i)=r(k) \geq 0$, we have another expression of Example 1.5 [5].

10. Fundamental theorem (I) of K. H. Look. In this section we shall give a neat but essentially equivalent proof of the fundamental theorem (I) given by K. H. Look [14] and an extension of this theorem using the minimum problem.

Proposition 10.1 (Fundamental theorem of Look). Let $D$ be a bounded schlicht domain and $f(z) \equiv\left(f_{1}(z), \ldots, f_{n}(z)\right)^{T}$ be any holomorphic mapping with the condition $|f(z)| \leq M$ in $D$, then we have

$$
(d f(z) / d z)^{*}(d f(z) / d z) \leq \|^{2} T_{D}(z, \bar{z}), \quad z \in D
$$

and

$$
\left|J_{f}(z)\right|^{2} \leq M^{2 n} \operatorname{det} T_{D}(z, \bar{z}), \quad z \in D .
$$

Proof. Let $M_{D}^{K(2)}(z, t)$ be the minimizing function with the condition $Q(z, \bar{z}) \equiv 0$ and $K(2)=\left(A_{1}, A_{2}\right)$, and $F(z)$ be a holomorphic mapping $k_{D}(z, \bar{t}) f(z) \in \mathcal{\complement}^{2}(D)$, then by (3.8), (3.9) and the Riesz's theorem for bounded linear functionals, we have

$$
\int_{D} F(z) \times M_{D}^{K(2) *}(z, t) \omega_{z}=f(t) A_{1}^{*}-f_{1}(t) T^{-1}\left(k_{10} A_{1}^{*} k^{-1}-A_{2}^{*}\right),
$$

where $f_{1}(t) \equiv D_{z} f(t)$ and $T \equiv T_{D}(t, \tau)$. Setting $\left(A_{1}, A_{2}\right)=\left(0, T_{D}(t, \tau)\right)$ (this is possible since $f(z)=T_{D}(t, T) z$ belongs to $\bigodot_{(0, T)}^{2}(D)$ ), we have

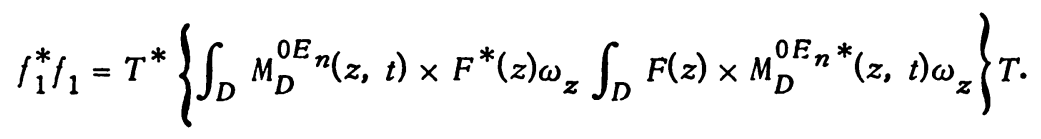

For an arbitrary $n \times 1$ vector $u$, we have, by the Schwarz inequality,

$$
\begin{aligned}
& u^{*} f_{1}^{*} f_{1} u \leq \int_{D}|F(z)|^{2} \omega_{z}\left(u^{*} T \int_{D} M_{D}^{0 E}{ }^{0} M_{D}^{0 E_{n} *} \omega_{z} T u\right) \\
& \leq k M^{2} u^{*} T(k T)^{-1} T u=M^{2} u^{*} T u \text {. }
\end{aligned}
$$


This shows (10.1) and the refore (10.2).

Theorem 10.1. Under the same hypothesis as in Proposition 10.1, we have

$$
f(z) f^{*}(z)+f_{1}(z) T_{D}^{-1}(z, \bar{z}) f_{1}^{*}(z) \leq M^{2} \times E_{n}, \quad z \in D,
$$

and

$$
\left|J_{f}(z)\right|^{2} \leq M^{2 n} \operatorname{det} T_{D}(z, \bar{z})\left(1-|f(z)|^{2} / M^{2}\right), \quad z \in D .
$$

If $f(z)$ belongs to $B H(D)$, we have

$f_{1}^{*}(z)\left\{E_{n}+f(z) f^{*}(z) /\left(M^{2}-|f(z)|^{2}\right)\right\} f_{1}(z) \leq M^{2} T_{D}(z, \bar{z}), \quad z \in D$.

Proof. In (10.3), setting $F(z)=k_{D}(z, \bar{t}) f(z)$, which belongs to $\mathcal{L}^{2}(D)$, and $\left(A_{1}, A_{2}\right)=(F(t), d F(t) / d z)$, we have

$$
\int_{D} F(z) \times M_{D}^{A_{1} A_{2} *}(z, t) \omega_{z}=k\left(f f^{*}+f_{1} T^{-1} f_{1}^{*}\right),
$$

where $k \equiv k_{D}(t, \tau)$. By a way similar to that of the proof of Proposition 10.1, we obtain

$$
k^{2}\left(f f^{*}+f_{1} T^{-1} f_{1}^{*}\right)^{2} \leq M^{2} k^{2}\left(f f^{*}+f_{1} T^{-1} f_{1}^{*}\right) .
$$

By the diagonalization of Hermitian matrices, we have (10.4) and thus (10.5) (cf. (10.1) and (10.2)).

Let us assume that $f(z)$ belongs to $B H(D)$ in (10.4). Since $f_{1} T^{-1} f_{1}^{*} \leq$ $M^{2} \times\left(E_{n}-f f^{*} / M^{2}\right)$ follows from (10.4), we obtain $f_{1}^{*}\left(E_{n}-f f^{*} / M^{2}\right)^{-1} f_{1} \leq$ $M^{2} T$ by taking the inverse on both sides of the above. If $A$ and $B$ are positive definite Hermitian matrices and satisfy $A \leq B$, we have $A^{-1} \geq B^{-1}$, because, from a known theorem of matrices, $A$ and $B$ are simultaneously brought to diagonal matrices with positive diagonal elements by operating suitable regular matrices $P^{*}$ and $P$ on each of $A$ and $B$ as $P^{*} A P$ and $P^{*} B P$. Noting that $\left(E_{n}-f f^{*} / M^{2}\right)^{-1}=E_{n}+f f^{*} /\left(M^{2}-|f|^{2}\right)$, we get (10.6), which is an extension of (10.1) for $f(z) \in B H(D)$.

\section{REFERENCES}

1. S. Bergman, Über die kernfunktion eines Bereiches und ihr Verhalten am Rande, J. Reine Angew. Math。 169 (1933), 1-42; ibid. 172 (1934), 89-128.

2. - Sur la fonction-noyau d'un domaine et ses application dans la théorie des transformations pseudoconformes, Mém. Sci. Math., no. 108, GauthierVillars, Paris, 1948. MR 11, 344.

3. - The kernel function and conformal mapping, 2nd ed., Math. Surveys, no. 5, Amer. Math. Soc., Providence, R. I., 1970.

4. B. L. Chalmers, On boundary behavior of the Bergman kernel function and related domain functionals, Pacific J. Math, 29 (1969), 243-250. MR 40 \#402. 
5. - Subspace kernels and minimum problems in Hilbert spaces with kernel function, Pacific J. Math. 31 (1969), 619-628.

6. B. A. Fuks, Über geodasische Mannigfaltigkeiten einer invarianten Geometrie, Mat. Sb. 2 (44) (1937), 567-594.

7. - Special chapters in the theory of analytic functions of several complex variables, Fizmatgiz, Moscow, 1963; English transl., Transl. Math. Monographs, vol, 14, Amer. Math. Soc., Providence, R. I., 1965. MR 30 \#4979; 32 \#5915.

8. - Ricci curvature of a Bergman metric invariant under biholomorphic mappings, Dokl. Akad. Nauk SSSR 167 (1966), 996-999= Soviet Math. Dokl. 7 (1966), 525-529. MR 33 \#4954.

9. L. Hörmander, $L^{2}$ estimates and existence theurems for the $\bar{\partial}$ operator, Acta Math. 113 (1965), 89-152. MR 31 \#3691.

10. L. K. Hua, On the estimation of the unitary curvature of the space of several complex variables, Sci, Sinica 4 (1955), 1-26.

11. S. Kato, Canonical domains in several complex variables, Pacific J. Math 21 (1967), 279-291. MR 35 \#5659.

12. K. Kikuchi, Canonical domains and their geometry in $C^{n}$, Pacific J. Math. 38 (1971), 681-696. MR $46 \# 3836$.

13. S. Kobayashi, Geometry of bounded domains, Trans. Amer. Math. Soc. 92 (1959), 267-290. MR 22 \#3017.

14. K. H. Look, Schwarz lemma and analytic invariants, Sci. Sinica 7 (1958), 453-504. MR 21 \#5028.

15. W. T. Martin, On a minimum problem in the theory of analytic functions of several variables, Trans. Amer. Math. Soc. 48 (1940), 351-357. MR 2, 86.

16. S. Matsuura, On the normal domains and the geodesics in the bounded symmetric spaces and the projective space, Sci. Rep. Gunma Univ. 15 (1966), 1-21.

17. - Bergman kernel functions and the three types of canonical domains, Pacific J. Math. 33 (1970), 363-384. MR 43 \#560.

18. H. S. Shapiro, Reproducing kernels and Beurling's theorem, Trans. Amer. Math. Soc. 110 (1964), 448-458. MR 28 \#2225.

19. J. M. Stark, Minimum problems in the theory of pseudoconformal transformations and their application to estimation of the curvature of the invariant metric, Pacific J. Math. 10 (1960), 1021-1038. MR 22 \#12237.

20. T. Tsuboi and S. Matsuura, Some canonical domains in $C^{n}$ and moment of inertia theorems, Duke Math. J. 36 (1969), 517-536. MR 41 \#509.

21. W. Wirtinger, Über eine Minimalaufgabe im Gebiete der analytischen Funktionen von mehreren Veränderlichen, Monatsh. Math. Phys. 47 (1939), 426-431. MR 1, 10 .

22. K. Yano and S. Bochner, Curvature and Betti numbers, Ann. of Math. Studies, no. 32, Princet on Univ. Press, Princeton, N. J., 1953. MR 15, 989.

DEPARTMENT OF MATHEMATICS, NAGOYA INSTITUTE OF TECHNOLOGY, NAGOYA, JAPAN 